\title{
A regime-switching copula approach to modeling day-ahead prices in coupled electricity markets
}

\author{
Anca Pircalabu ${ }^{* \dagger}$ and Fred Espen Benth ${ }^{\S}$
}

\begin{abstract}
The recent price coupling of many European electricity markets has triggered a fundamental change in the interaction of day-ahead prices, challenging additionally the modeling of the joint behavior of prices in interconnected markets. In this paper we propose a regime-switching AR-GARCH copula to model pairs of day-ahead electricity prices in coupled European markets. While capturing key stylized facts empirically substantiated in the literature, this model easily allows us to 1) deviate from the assumption of normal margins and 2) include a more detailed description of the dependence between prices. We base our empirical study on four pairs of prices, namely Germany-France, Germany-Netherlands, Netherlands-Belgium and Germany-Western Denmark. We find that the marginal dynamics are better described by the flexible skew $t$ distribution than the benchmark normal distribution. Also, we find significant evidence of tail dependence in all pairs of interconnected areas we consider. As a first application of the proposed empirical model, we consider the pricing of financial transmission rights, and highlight how the choice of marginal distributions and copula impacts prices. As a second application we consider the forecasting of tail quantiles, and evaluate the out-of-sample performance of competing models.
\end{abstract}

Keywords: Day-ahead electricity prices; Market coupling; Copula models; Tail dependence; Financial transmission rights; Tail quantile forecasting.

\section{Introduction}

Since the many projects launched over the past decade to achieve increased market integration across day-ahead electricity markets in Europe, the day-ahead electricity price convergence between market areas has increased significantly, adding to the complexity of modeling the joint behavior of day-ahead electricity prices.

The first significant step towards market integration in Europe was taken with the introduction of the Nord Pool market, which is the main platform for trading power in most Nordic and Baltic countries. At Nord Pool, the bidding areas are price coupled, meaning that the transmission capacity is auctioned as an implicit part of the day-ahead auction of electrical energy. This achieves - as opposed to the explicit auctioning, where the transmission capacity auction and the day-ahead auction of electrical energy are separate actions - the efficient utilization of interconnectors and results in smaller price differences between the bidding areas. Since Nord Pool's creation in the mid 90's, many countries have joined and Nord Pool comprises today Norway, Sweden, Denmark, Finland, Estonia, Latvia and Lithuania. For the sake of completion, we also mention that Nord Pool has taken sole ownership of the UK market as of 2014.

Although Nord Pool has a long-standing history of price coupling, such initiatives are more recent for other European countries. In 2006, the so-called Tri-Lateral Market Coupling (TLC) project comprising France, Belgium and the Netherlands was initiated. Later in 2010, the price coupling of Central West Europe (CWE) was achieved, integrating Germany, France, Austria, Belgium, the Netherlands and Luxembourg. In 2014, a crucial milestone was reached with the price coupling of the North Western European (NWE) region, covering

\footnotetext{
${ }^{*}$ Corresponding author. E-mail: anca@math.aau.dk

$\dagger$ Department of Mathematical Sciences, Aalborg University, Fredrik Bajers Vej 7G, 9220 Aalborg Øst, Denmark.

${ }^{\ddagger}$ Department of Quantitative Analytics, Neas Energy, Skelagervej 1, 9000 Aalborg, Denmark.

$\S$ Department of Mathematics, University of Oslo, PO Box 1053 Blindern, N-0316 Oslo, Norway, fredb@math.uio.no.
}

Date: 14 September 2017.

Anca Pircalabu is supported by the Innovation Fund Denmark. Fred Espen Benth acknowledges support from FINEWSTOCH, funded by the Norwegian Research Council. The authors would like to thank Jesper Jung, Esben Høg and Thomas Aalund Fredholm for providing valuable comments and suggestions. Two anonymous referees are also thanked for their constructive criticism and suggestions, which improved the presentation of this paper. 
the CWE region, the UK, the Nordic and Baltic countries - and as of 2016, the price coupling region has grown to include 19 European countries. Also, a new algorithm regarding how cross-border capacities are included in the day-ahead price calculation was introduced on 20 May 2015 in the CWE region, with the transition from the Available Transmission Capacity (ATC) methodology to the more efficient Flow-Based (FB) methodology.

For the joint modeling of day-ahead electricity prices in two interconnected areas, price coupling has induced a fundamental change that cannot be ignored. In the case of the CWE region for example, the transition from explicit to implicit auctioning of transmission capacity has meant that equal prices in two adjacent markets are very often observed now. As already accentuated in the existing literature (see e.g. Füss et al. (2013, 2015)), this has generally resulted in the non-feasibility of previously proposed models. For one, the pricing of spread options such as the financial transmission rights cannot be achieved using spread option formulas á la Margrabe. Also in risk management applications, the classical reduced-form models are no longer suitable for modeling the joint behavior of prices. To exemplify some events and their influence on the day-ahead electricity prices, we plot in Fig. 1 the evolution of the German-French price spread at hour 8, for the period 1 January 2009 to 25 September 2016. According to Fig. 1, the most visible change in the spread dynamics was caused by the price coupling of Germany and France in 2010, leading to an increasing amount of exact price convergence in the following period.

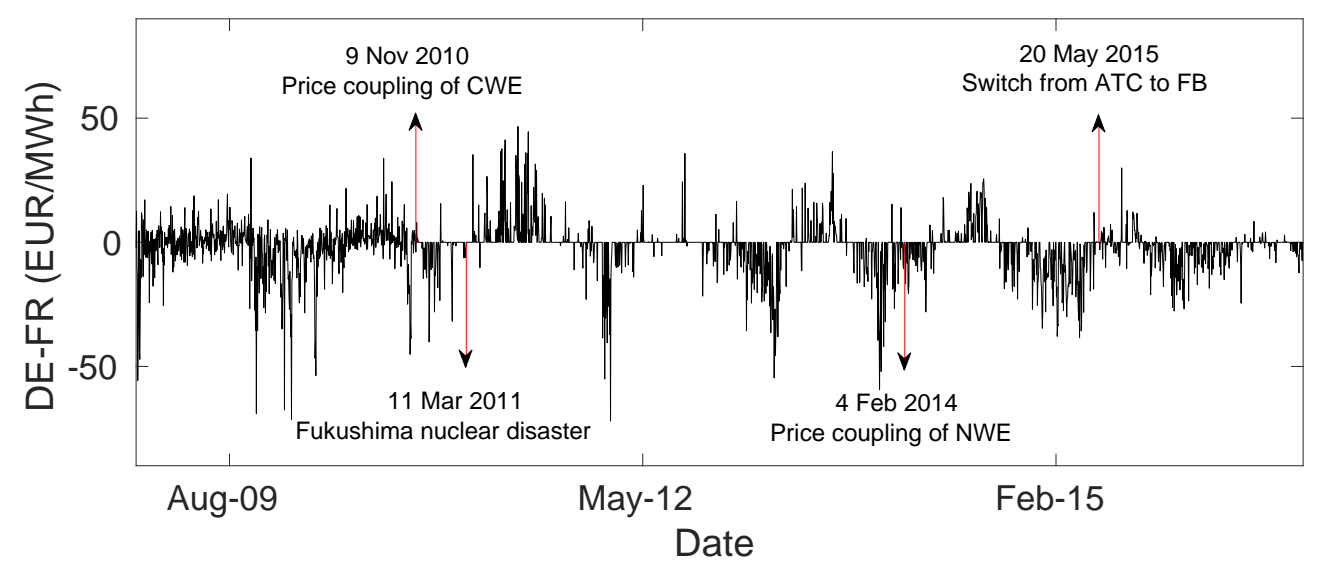

Figure 1: Day-ahead electricity price spread for Germany-France (DE-FR) at hour 8, from 1 January 2009 to 25 September 2016.

In this paper, we propose a regime-switching AR-GARCH copula model for the joint behavior of pairs of day-ahead prices in coupled electricity markets, inspired by ideas from Haldrup and Nielsen (2006) and Haldrup et al. (2010). The regime-switching part is essential for distinguishing the case of identical prices from the case of non-identical prices. The AR-GARCH functions as a filter to remove the serial dependence in the conditional means and conditional variances. The use of copula models facilitates the analysis in the sense that marginal models for day-ahead prices are treated separately from the dependence structure. Furthermore, copulas allow for the easy transition to more realistic distributional assumptions for the residuals, and also allow for the inclusion of a more detailed description of the dependence structure when the prices do not coincide. Our empirical study concentrates on four coupled markets: Germany-France, Germany-Netherlands, Netherlands-Belgium and Germany-Western Denmark.

The proposed model has a wide range of applications in the context of derivatives pricing, hedging and risk management. Here, we consider two applications: The pricing of financial transmission rights, and the out-ofsample forecasting of tail quantiles. In both applications, we study how the results are impacted by the choice of marginal distributions and the choice of copula. Furthermore in the forecasting exercise, we demonstrate the usefulness of the proposed modeling framework by comparing the forecast performance of copula-based models against a simple alternative that is not copula-based.

The existing literature on bivariate or multivariate models for day-ahead prices in coupled electricity markets is scarce. Due to the aforementioned increasing complexity in the joint price dynamics, many authors employ fundamental models, with some examples being Füss et al. $(2013,2015)$ and Kiesel and Kustermann (2016). In Füss et al. (2013, 2015), besides providing a thorough institutional background and a description of the advances regarding market coupling in Europe and the US, the authors propose a fundamental model for the day-ahead electricity prices in interconnected markets, focusing on the pricing of transmission rights as an application. Furthermore in Füss et al. (2015), the authors provide a closed-form pricing formula for transmission rights, together with a sensitivity analysis. Kiesel and Kustermann (2016) propose a fundamental model in the spirit of Carmona et al. (2013), and also focus on the pricing aspect as an application, deriving closed-form futures 
and option prices. Moreover, they provide an empirical application using data from the German and French markets.

However appealing fundamental models are when it comes to their adaptability to changes in the market structure - which indeed happen quite often - they can be extremely data-heavy and thus both theoretically and empirically challenging, as also acknowledged by Kiesel and Kustermann (2016). In the empirical analysis of Kiesel and Kustermann (2016) regarding Germany-France, the data input amounts to many different time series: Day-ahead electricity price data, coal, gas and oil data to obtain a proxy for the marginal fuel, residual expected demand data for both countries (which is in turn obtained by subtracting renewable electricity generation from the expected demand), and also data on installed transmission capacities. Our proposed model on the other hand, which belongs to the class of reduced-form models, is not nearly as data intensive, while still being able to comply with the institutional framework.

Examples on applications of time series models in the context of price coupled electricity markets are Haldrup and Nielsen (2006), where a regime-switching multiplicative SARFIMA model is proposed for the modeling of price spreads in the Nord Pool area, and Haldrup et al. (2010), where a vector autoregressive model with long memory and regime switches is proposed and applied again to Nord Pool data.

In the context of copula models and their applications in energy markets, copulas have been gaining more interest recently. Some examples are Börger et al. (2009), who use copulas in the context of cross-commodity risk management, Benth and Kettler (2011), who employ copulas to model the spark spread, Avdulaj and Barunikl (2015), who use dynamic copulas to investigate oil-stock diversification, and Grothe and Schnieders (2011) and Elberg and Hagspiel (2015), who consider copulas in the context of wind power in Germany.

The remaining of this paper is organized as follows: Section 2 describes the data that our analysis is based on. Section 3 introduces the regime-switching AR-GARCH copula model. In Section 4 the proposed model is fitted to the data. Sections 5 and 6 present two empirical applications of the model. Section 7 concludes.

\section{Data}

Our empirical study relies on day-ahead electricity price data from four price coupled markets: Germany-France (DE-FR), Germany-Netherlands (DE-NL), Netherlands-Belgium (NL-BE), and Germany-Western Denmark (DE-DK1). After careful considerations, we choose to fix the sample window to the period 1 May 2011 to 20 May 2015. Since areas of CWE are mostly represented in our analysis, and we wish to analyze the period following price coupling, a natural starting point for the sample is 10 November 2010, which marks the first day of price coupling in the CWE region ${ }^{\dagger}$. Shortly after however, the Fukushima nuclear disaster followed, leading to a sudden change in German policy, with the closure of nuclear power plants and the significant focus on the development of renewable generation sources. To exclude the immediate perturbation caused by the Fukushima accident, we thus let the start of our sample be 1 May 2011. Regarding the end point, we choose the date marking the switch to the FB methodology. According to EPEX (2015), the FB coupling algorithm allows for more sophisticated grid modeling to optimize the flows on interconnectors in comparison to the ATC methodology. Effects of the change regarding how flows are included in the calculation of day-ahead prices are not evident from just looking at time series plots of the data. However, we do suspect some implications on the joint price dynamics, and let the sample end at this date as a result. We will get back to this issue later in the paper.

Having fixed the sample window to the period 1 May 2011 - 20 May 2015, we avoid the most significant structural changes in the institutional framework for the pairs belonging to the CWE region. Careful attention is however needed in the case of the DE-DK1 pair, where price coupling was first introduced on 4 February 2014. Before then, Western Denmark was volume coupled to Germany, using explicit auctioning. This is not necessarily as worrying as the case of price coupling in the CWE region, since exact price convergence between Germany and Western Denmark was achieved frequently also before their price coupling. Nevertheless, we shall treat the pair DE-DK1 with special consideration before applying the same modeling framework to all pairs of prices. Although the pair DE-DK1 complicates our analysis somewhat, its inclusion is motivated by having one of Nord Pool's bidding areas represented in our analysis, and also by having a pair where the in-feed of renewable energy is very significant in both markets.

We consider hourly price data separately for each hour of the day - so for all four pairs, we will have 24 hourly time series, consisting of 1,481 observations each. Fig. 2 shows time series plots of the four pairs of spreads at four different hours of the day, and Table 1 provides summary statistics in order to get a better sense of the data. To avoid distortions, few extreme prices have been truncated: Specifically, prices below -50 EUR/MWh were set to -50 , and prices above $150 \mathrm{EUR} / \mathrm{MWh}$ were set to $150 .^{\ddagger}$

\footnotetext{
$\dagger$ We note that price coupling in CWE was launched 9 November 2010, for delivery day 10 November 2010.

${ }^{\ddagger}$ For most hourly price time series no truncation is necessary, since prices below -50 or above 150 never occur. The maximum number of observations below -50 that are truncated in one single hourly price series is 7 , and corresponds to an hourly price series
} 
The spreads show a rather different behavior across pairs and also across the hours of the day. For DE-FR, we observe a changing seasonal pattern during (or near) off-peak. During summer off-peak, Germany usually imports electricity from France due to low renewables generation in Germany (less wind during summer periods, and very little solar during off-peak) and excess nuclear production in France due to the lower demand. During winter peak and off-peak, France usually imports from Germany due to increased demand and higher production in Germany caused by increased renewables generation.

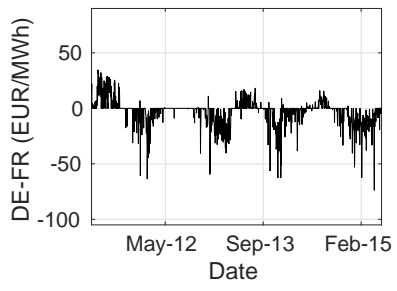

(a) Hour 2

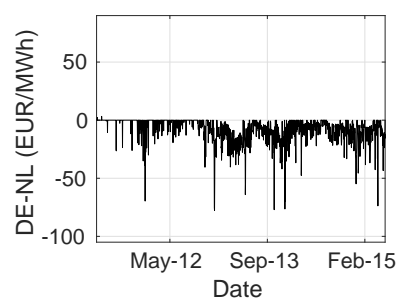

(e) Hour 2

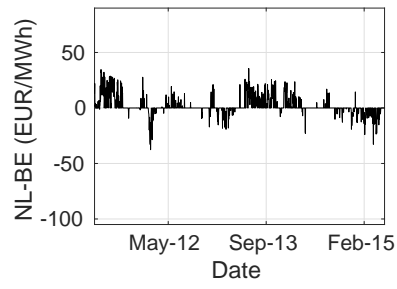

(i) Hour 2

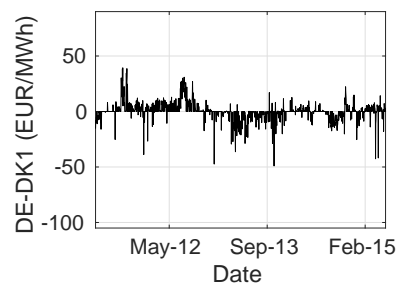

(m) Hour 2

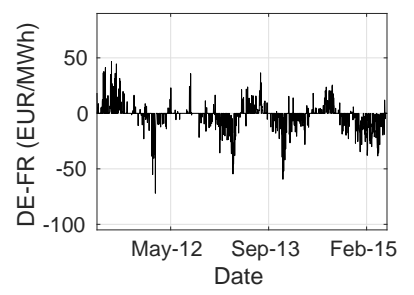

(b) Hour 8

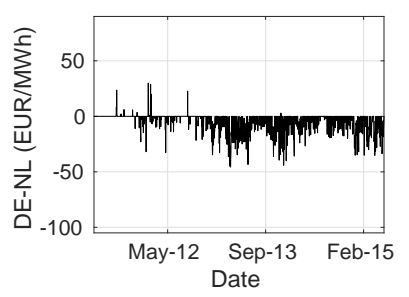

(f) Hour 8

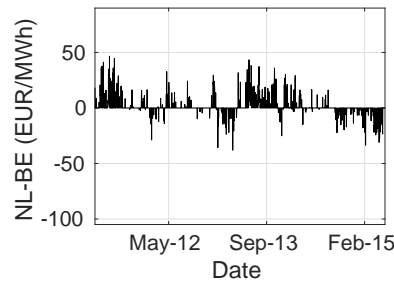

(j) Hour 8

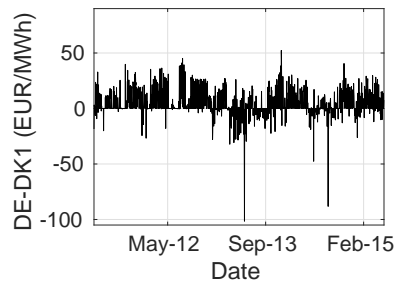

(n) Hour 8

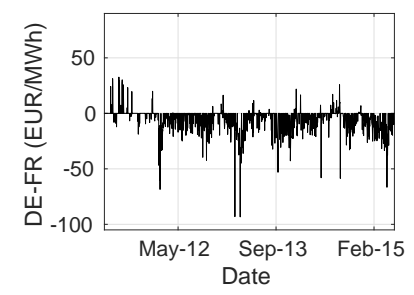

(c) Hour 14

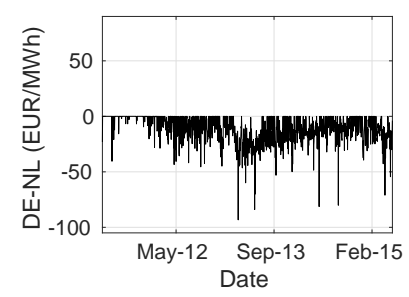

(g) Hour 14

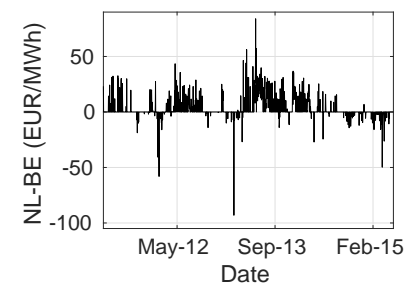

(k) Hour 14

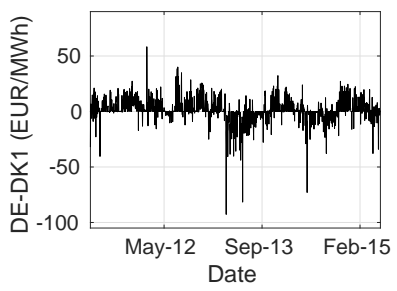

(o) Hour 14

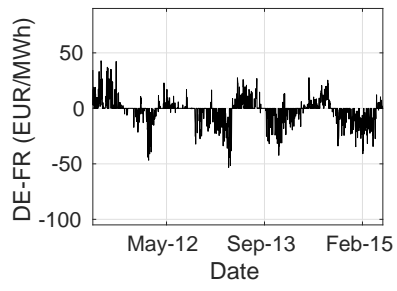

(d) Hour 20

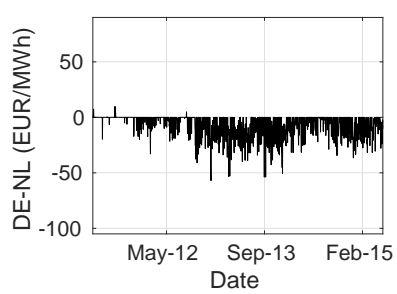

(h) Hour 20

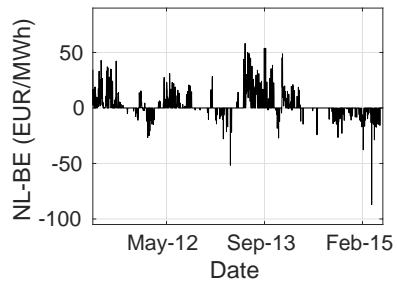

(l) Hour 20

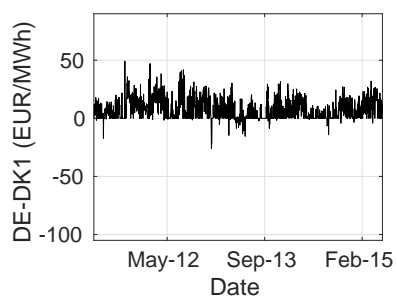

(p) Hour 20

Figure 2: Historical hourly spreads between the pairs DE \& FR, DE \& NL, NL \& BE and DE \& DK1 at four different hours of the day.

In the case of the DE-NL pair, the German price is almost always below the Dutch price, signaling Netherlands to be a significant importer of German power. This relation can be explained by the electricity generation mix in the Netherlands, which relies heavily on more expensive natural gas.

For NL-BE we see that the Dutch price is more often observed to be above the Belgian price, which again is due to the more expensive generation sources in the Netherlands compared to Belgium. However, both countries are mainly importers, and their day-ahead electricity prices are heavily influenced by conditions in Germany and France. A seasonal pattern is also observed here, but is not as persistent as in the case of DE-FR. According to TenneT (2013), this is because higher demand for electrical heating in France during winter is somehow reflected in the Belgian price, which in such situations would typically increase above the level of Dutch prices.

Regarding the DE-DK1 pair, prices in Western Denmark are usually below Germany. Renewable power is significant in both countries - DK1 has a lot of wind and DE has both wind and solar - however Denmark is connected to both Sweden and Norway, from where it can import cheap hydropower. A generation deficit

for DE. The maximum number of observations above 150 that are truncated in one single hourly price series is 6 , and corresponds to an hourly price series for FR. The truncation rule is mostly introduced to deal with cases like e.g. the extreme spikes we observe in DK1 on 7 June 2013, where prices reached approx. 2,000 EUR/MWh for five consecutive morning hours. 
typically takes place during summer periods in DK1 due to less wind. Depending on transmission capacity limitations, weather conditions, etc., imports come from Germany, Sweden and/or Norway.

Considering in more detail the summary statistics presented in Table 1, we see that exact price convergence or equivalently a price spread of zero occurs very often for all pairs and hours of the day. Moreover, the exact price coupling percentage can vary quite a lot depending on the hour of the day, and so can the mean and the standard deviation. We also observe a strong positive relation between the prices conditional on the spread being nonzero. This is of course expected due to the limited transmission capacity which impedes exact price convergence at all times - note that only a utilization of the available interconnector capacity below $100 \%$ would allow exact price convergence.

\begin{tabular}{|c|c|c|c|c|c|c|c|}
\hline & Spread $=0$ & Spread $>0$ & Spread $<0$ & Mean & Std. dev. & Lin. corr. & $\begin{array}{r}\text { Lin. corr. } \\
(\text { Spread } \neq 0)\end{array}$ \\
\hline \multicolumn{8}{|c|}{ Germany and France (DE - FR) } \\
\hline Hour 2 & $51.11 \%$ & $14.32 \%$ & $34.57 \%$ & -3.41 & 11.53 & 0.53 & 0.39 \\
\hline Hour 8 & $57.60 \%$ & $15.67 \%$ & $26.73 \%$ & -1.78 & 10.27 & 0.85 & 0.70 \\
\hline Hour 14 & $49.90 \%$ & $4.25 \%$ & $45.85 \%$ & -5.28 & 10.22 & 0.80 & 0.68 \\
\hline Hour 20 & $49.29 \%$ & $20.05 \%$ & $30.66 \%$ & -1.96 & 10.86 & 0.70 & 0.55 \\
\hline \multicolumn{8}{|c|}{ Germany and Netherlands (DE - NL) } \\
\hline Hour 2 & $35.11 \%$ & $0.14 \%$ & $64.75 \%$ & -7.96 & 10.38 & 0.48 & 0.43 \\
\hline Hour 8 & $58.41 \%$ & $1.08 \%$ & $40.51 \%$ & -4.69 & 8.29 & 0.88 & 0.81 \\
\hline Hour 14 & $34.77 \%$ & $0.00 \%$ & $65.23 \%$ & -10.79 & 11.90 & 0.67 & 0.55 \\
\hline Hour 20 & $50.17 \%$ & $0.54 \%$ & $49.29 \%$ & -6.96 & 9.90 & 0.63 & 0.49 \\
\hline \multicolumn{8}{|c|}{ Netherlands and Belgium (NL - BE) } \\
\hline Hour 2 & $65.83 \%$ & $21.00 \%$ & $13.17 \%$ & 1.50 & 7.71 & 0.72 & 0.57 \\
\hline Hour 8 & $71.10 \%$ & $17.89 \%$ & $11.01 \%$ & 1.17 & 7.59 & 0.90 & 0.79 \\
\hline Hour 14 & $69.82 \%$ & $21.54 \%$ & $8.64 \%$ & 2.75 & 9.60 & 0.75 & 0.58 \\
\hline Hour 20 & $66.51 \%$ & $21.81 \%$ & $11.68 \%$ & 1.92 & 9.33 & 0.70 & 0.36 \\
\hline \multicolumn{8}{|c|}{ Germany and Western Denmark (DE - DK1) } \\
\hline Hour 2 & $36.60 \%$ & $35.72 \%$ & $27.68 \%$ & 0.09 & 7.90 & 0.76 & 0.57 \\
\hline Hour 8 & $30.11 \%$ & $55.10 \%$ & $14.79 \%$ & 4.75 & 10.99 & 0.79 & 0.68 \\
\hline Hour 14 & $28.83 \%$ & $46.58 \%$ & $24.58 \%$ & 1.47 & 10.11 & 0.78 & 0.70 \\
\hline Hour 20 & $23.63 \%$ & $71.51 \%$ & $4.86 \%$ & 7.76 & 9.24 & 0.71 & 0.69 \\
\hline
\end{tabular}

Table 1: Summary statistics for the spread between day-ahead electricity prices for four selected periods. The hour denoted "Hour 2" represents the hour starting at 2 and ending at 3, and the same holds for all other hours. Furthermore, the time zone is CET. The sample covers the period 1-May-2011 to 20-May-2015, a total of 1,481 observations in each of the four time series per spread we consider here. All spreads are measured in EUR/MWh.

In Fig. 3, we consider in more detail the behavior of exact price convergence across the hours of the day. For the CWE, there is generally less exact price convergence during the night hours, and also during midday. For DE-DK1, the situation is somewhat reversed, with more coupling during off-peak.

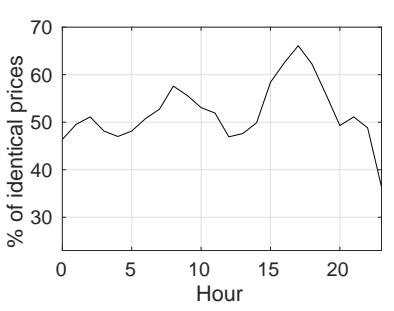

(a) DE \& FR

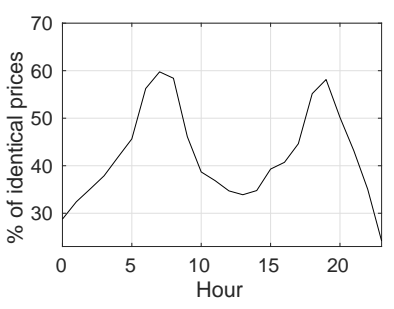

(b) DE \& NL

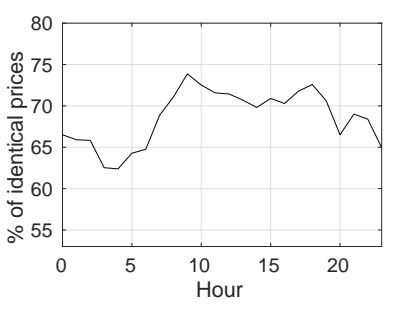

(c) NL \& BE

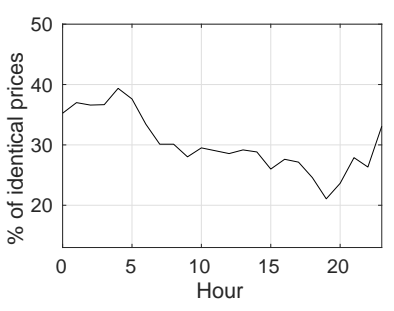

(d) DE \& DK1

Figure 3: Percentages of absolute price convergence grouped by hour of the day.

The shape of the plots for the CWE areas, especially the plot for DE-NL, where the shape is most pronounced, reminds us of the "two hump" behavior of German day-ahead prices. Typically, the German price is low during the night because of lower demand and high wind in-feed (especially during winter). Prices are also lowered during midday, due to the high solar in-feed, which peaks at noon. Why the shape of German prices during the day is reflected in the absolute coupling percentage can, to a high extent, be explained by the mix of generation assets across markets. 
Let us consider the example of DE-NL. The shape of the Dutch price during the peak hours is rather flat compared to Germany, and the level of prices is higher due to the more expensive marginal cost of natural gas-fired power plants, which play a major role in the daily fuel mix. A smaller price difference between DE-NL is thus more likely to occur during morning and evening, when the German prices reach their highest level. This implies that the remaining gap that needs to be closed in order to obtain exact price convergence is smaller during these periods, and more likely to be achieved with less than a $100 \%$ utilization of the available transmission capacity.

In the case of DE-DK1, the increased exact price convergence during off-peak can be explained by the combination of lower demand and high in-feed of wind generation in both countries, which leads to smaller price differences during off-peak.

\section{Model specification}

To model the evolution of prices in two interconnected markets, we propose a regime-switching AR-GARCH copula model for each hour of the day separately. We allow for two regimes, which are in fact observable and can be categorized as 1) identical and 2) non-identical prices. Making this distinction is of uttermost importance since it allows us to replicate periods of spreads equaling zero (see Fig. 2 and Table 1). ${ }^{\dagger}$

Our modeling procedure can be divided into three steps: In a first step, we deal with the seasonality aspect. For all price areas we consider in this paper, that is BE, DK1, DE, FR and NL, the mechanism of day-ahead price formation is based on matching supply and demand curves on an hourly basis. Hence, the strong seasonal variation that characterizes both the demand and the supply is reflected in the behavior of the electricity prices. To correct the data for seasonality, suitable seasonal functions are applied to each pair of prices, for each hour of the day. The seasonal functions are such that the identical/non-identical price patterns are preserved in the deseasonalized prices.

In a second step, regime-switching AR-GARCH filters are applied to each pair of deseasonalized prices. Again in this case, the resulting residuals will maintain the same pattern of identical and non-identical prices that we observe in the actual price data. Hence, the residuals can be split according to 1) a case of perfect positive dependence, where the residuals from the regime-switching AR-GARCH will coincide, and 2) a case of non-perfect dependence.

In a third step, we study the dependence of non-equal residuals by applying the flexible copula models. Below, we elaborate on the second and third steps in our modeling framework.

\subsection{A regime-switching AR-GARCH copula model}

Let us denote by $y_{n, t, i}$ the price in area $i$, at hour $n$ and day $t$, which has been corrected for deterministic seasonality. Since we only consider pairs of day-ahead prices, $i=1,2$. To simplify the notation in what follows, we suppress the subscript $n$. Whenever we refer to $y_{t, i}$, we mean the deseasonalized day-ahead price for some given hour of the day. We consider the following dynamics for the prices: If $y_{t, 1}=y_{t, 2}$, then we are in the regime of equal prices denoted by $s_{t}=0$, and for $i=1,2$,

$$
\begin{aligned}
y_{t, i} & =\sum_{p=1}^{P} \phi_{p}^{(0)} \frac{1}{2}\left(y_{t-p, 1}+y_{t-p, 2}\right)+\epsilon_{t, i} \\
\epsilon_{t, i} & =\sigma_{t, i} \eta_{t, i} \\
\sigma_{t, i}^{2} & =\omega^{(0)}+\alpha^{(0)} \frac{1}{2}\left(\epsilon_{t-1,1}^{2}+\epsilon_{t-1,2}^{2}\right)+\beta^{(0)} \frac{1}{2}\left(\sigma_{t-1,1}^{2}+\sigma_{t-1,2}^{2}\right) .
\end{aligned}
$$

Note that under $s_{t}=0$, it follows from the above that $\epsilon_{t, 1}=\epsilon_{t, 2}$ and $\sigma_{t, 1}^{2}=\sigma_{t, 2}^{2}$. If $y_{t, 1} \neq y_{t, 2}$, then we are in the regime of non-equal prices denoted by $s_{t}=1$, and for $i=1,2$,

$$
\begin{aligned}
y_{t, i} & =\sum_{p=1}^{P} \phi_{p}^{(i)} y_{t-p, 1}+\sum_{q=1}^{Q} \xi_{q}^{(i)} y_{t-q, 2}+\epsilon_{t, i} \\
\epsilon_{t, i} & =\sigma_{t, i} \eta_{t, i} \\
\sigma_{t, i}^{2} & =\omega^{(i)}+\alpha^{(i)} \epsilon_{t-1, i}^{2}+\beta^{(i)} \sigma_{t-1, i}^{2} .
\end{aligned}
$$

According to Eqs. (1) and (4) for the conditional mean, and Eqs. (3) and (6) for the conditional variance, all parameters vary depending on the regime. We shall thus obtain three sets of parameter estimates: One set

\footnotetext{
${ }^{\dagger}$ We mention in passing that univariate time series models of the ARMA-GARCH type have been successfully applied to model day-ahead electricity prices previously (see e.g. Keles et al. (2012) and Paraschiv (2013)).
} 
corresponding to the regime where prices are identical, denoted with superscript (0), and two sets of parameters corresponding to the regime where prices are not identical, denoted with superscripts $(i)$, for $i=1,2$.

To provide a parsimonious representation, the structure of the models is slightly different under the two regimes. In the model for the conditional mean, identical prices are explained by lagged average prices from the two areas, whereas no averaging appears in the case of non-identical prices. We note that aside from autoregressive terms, we allow for cross-equation effects by including lagged values of "the other price series", in the spirit of vector autoregressions. Nevertheless, we will refer to the model for the conditional mean as an AR. The model for the conditional variance resembles the $\operatorname{GARCH}(1,1)$ specification: In times of identical prices, the model contains averages of lagged values, whereas the non-identical price regime is characterized by the classical GARCH(1,1) dynamics. While accounting for serial dependence and heteroskedastisity, we stress that the construction of the model in Eqs. (1)-(6) ensures that the regime switching nature of prices is maintained in the standardized residuals.

For the distribution of the standardized residuals denoted by $\eta$ in Eqs. (2) and (5), we consider different possibilities. First, we consider the standard choice of a normal distribution:

$$
\eta_{t, i} \sim \mathrm{N}(0,1) \quad \text { for } i=1,2
$$

To allow for more flexibility, we also consider

$$
\eta_{t, i} \sim \begin{cases}\text { Skew } t\left(0,1, \nu^{(0)}, \lambda^{(0)}\right) & \text { if } s_{t}=0 \\ \text { Skew } t\left(0,1, \nu^{(i)}, \lambda^{(i)}\right) & \text { if } s_{t}=1, \text { for } i=1,2,\end{cases}
$$

where $\nu$ and $\lambda$ denote the degrees of freedom and skewness parameters of the skew $t$ distribution, respectively, and are allowed to vary with the regimes.

Next, we introduce copulas to model the dependence structure of standardized residuals belonging to the non-equal price regime. Copulas are flexible tools that enable the study of dependence structures beyond the linear correlation. For the two-dimensional case we consider here, a copula is essentially a bivariate distribution function $C$ defined on the unit cube, with standard uniform margins. The central result concerning copulas is due to Sklar's theorem [Sklar (1959)], which states that if we assume $\left(\eta_{1}, \eta_{2}\right)$ to have bivariate distribution function $F$ and univariate marginal distribution functions $F_{1}, F_{2}$, then there exists a bivariate copula $C$, such that

$$
F\left(\eta_{1}, \eta_{2}\right)=C\left(F_{1}\left(\eta_{1}\right), F_{2}\left(\eta_{2}\right)\right)
$$

If the marginal distribution functions are continuous, we have furthermore that the copula is unique, and can be defined through

$$
C\left(u_{1}, u_{2}\right)=F\left(F_{1}^{-1}\left(u_{1}\right), F_{2}^{-1}\left(u_{2}\right)\right),
$$

where the $u$ 's represent standard uniform variables and the $F^{-1}$ 's represent the inverse of the marginal distribution functions. Adding to the flexibility of copulas is that the converse of Sklar's theorem also holds, meaning that we can combine two marginal distributions, be it from different families even, to form a joint distribution.

Since we condition on the information generated by past observations of the variables (by using AR-GARCH filters), we are in fact considering here the conditional copula. Also in this case, Sklar's theorem applies, as shown in Patton (2001).

\subsection{Estimation procedure}

Estimation of the model parameters is done by maximum likelihood. The joint conditional density function is obtained by differentiating Eq. (9), and thus the full-sample log-likelihood takes the form

$$
\log \mathcal{L}=\sum_{i=1}^{2} \sum_{t=1}^{T} \log f_{i}\left(y_{t, i} \mid \mathcal{F}_{t-1} ; \Theta_{i}\right)+\mathbb{I}_{s_{t}=1} \sum_{t=1}^{T} \log c\left(\left(u_{t, 1}, u_{t, 2}\right) \mid \mathcal{F}_{t-1} ; \Theta_{3}\right),
$$

where

$$
\sum_{t=1}^{T} \log f_{i}\left(y_{t, i} \mid \mathcal{F}_{t-1} ; \Theta_{i}\right)=\mathbb{I}_{s_{t}=0} \sum_{t=1}^{T} \log f_{i}\left(y_{t, i} \mid \mathcal{F}_{t-1} ; \Theta_{i}^{(0)}\right)+\mathbb{I}_{s_{t}=1} \sum_{t=1}^{T} \log f_{i}\left(y_{t, i} \mid \mathcal{F}_{t-1} ; \Theta_{i}^{(i)}\right) .
$$

In Eqs. (11) and (12), the functions $f_{i}$ denote the conditional marginal density functions for area 1 and 2 , and $c$ is the conditional copula density representing the non-equal price regime. The variable $\mathbb{I}_{\Omega}$ denotes the indicator function of the event $\Omega$. Furthermore, $\mathcal{F}_{t-1}$ denotes the filtration, and $\left(\Theta_{1}, \Theta_{2}, \Theta_{3}\right)$ refer to the parameters for the full model. Specifically, $\left(\Theta_{1}, \Theta_{2}\right)$ refer to the parameters of the regime-switching AR-GARCH model, and $\Theta_{3}$ refers to the copula parameters. 
Recall that by construction of the model, $u_{t, 1}=u_{t, 2}$ if $y_{t, 1}=y_{t, 2}$, and $u_{t, 1} \neq u_{t, 2}$ otherwise. In the equal price regime, the residuals thus coincide and we have a case of perfect positive dependence (also referred to as the Fréchet-Hoeffding upper bound), where there is no copula parameter to be estimated. Hence, the only copula parameter to be estimated corresponds to the pair of standardized residuals in the non-equal state.

Usually, the model parameters are obtained by performing a multi-stage maximum likelihood estimation, where the marginal models and the copula are considered separately, see e.g. Dias and Embrechts (2009) and Patton (2013). Since the parameter vectors of the marginal models have common elements in our case, these cannot be estimated separately. Hence, in a first step, a joint numerical maximization of the the first term in Eq. (11) is performed to obtain estimates for $\left(\Theta_{1}, \Theta_{2}\right)$. Then, standardized residuals are obtained, and the uniforms entering the copula log-likelihood follow immediately from applying the probability integral transform. In a second step, the copula parameters are estimated by maximizing the copula log-likelihood numerically.

\section{Model fitting}

\subsection{Seasonal function}

As mentioned in Section 3, the first step in our data analysis is concerned with the seasonality correction. Following the related literature (see e.g. Lucia and Schwartz (2002), Haldrup et al. (2010) and Janczura et al. (2013)), we consider the following seasonal function $\Lambda$ for each area in a price pair:

$$
\Lambda_{t}^{s_{t}}=a^{s_{t}}+b^{s_{t}} t+c^{s_{t}} \sin (2 \pi t / 365)+d^{s_{t}} \cos (2 \pi t / 365)+\sum_{j=1}^{4} w_{j}^{s_{t}} W_{t}^{j}+h^{s_{t}} H_{t}, \quad \text { for } s_{t}=0,1 .
$$

In Eq. (13), $a$ is a constant, $b$ denotes the trend coefficient and $c$ and $d$ are coefficients for the yearly cycle. To capture the weekly seasonality, we use day-of-week dummies denoted by $W^{j}$, with their corresponding coefficients denoted by $w_{j}$. We group Tuesday, Wednesday and Thursday in one dummy variable, and have therefore limited ourselves to only four day-of-week coefficients. Lastly, the dummy variable $H$ is included to capture holiday effects, and its coefficient is denoted by $h .^{\dagger}$

The seasonal functions are fitted to the data by ordinary least squares. To ensure that the deseasonalized price data corresponding to a given pair retains the same regime-switching structure, note that we let all parameters vary with the regime $s_{t}$. For a given pair, the parameter estimates corresponding to the state $s_{t}=0$, i.e., state of equal prices, will by construction be the same for area $i=1$ and area $i=2$. For the state $s_{t}=1$, i.e., state of non-equal prices, the parameter estimates will differ for the two areas.

\subsection{Marginal models}

After removing the deterministic seasonal component, the regime-switching AR-GARCH model introduced in Eqs. (1) - (6) is fitted to the data. Specifically, the model is fitted to all four pairs of prices and all hours of the day, which corresponds to a total of 96 models. For the order of the autoregression $(P)$ and cross-equation effects $(Q)$ in the equations for the conditional mean, we consider $P=1, \ldots, 7$ and $Q=1, \ldots, 7$. The optimal order of the models is chosen based on the Bayesian Information Criterion (BIC). In the interest of brevity, we provide the detailed results in Appendix A, Table 12. We do however mention that the order of $Q$ is zero for the majority of the models, revealing that the cross-equation effects (cf. Eq. (4)) can generally be omitted.

Model specifications other than those stated in Eqs. (1) - (6) were experimented with, and we note that our proposed regime-switching AR-GARCH model is one of many possible specifications. We found however our specification to be superior in terms of parsimony and its fit to the data.

To provide some evidence for the fit of the regime-switching AR-GARCH, we consider as an example the DE-FR pair at hour 8. Table 2 presents the estimation results, and Fig. 4 displays sample autocorrelation and quantile plots. Sample autocorrelation plots are provided for both the standardized residuals and the squared standardized residuals, and give indication of almost no serial correlation left. Regarding the quantile plots, we provide both the fit with the normal and the skew $t$ distribution, illustrating the superiority of the latter. Similar results to those in Fig. 4 were obtained for the remaining models. In Appendix A, we provide some additional results for the remaining pairs at hour 8 .

\subsection{The pair DE-DK1}

Before proceeding to copula modeling, we recall that the pair DE-DK1 behaved differently compared to the other pairs in terms of initiatives that impacted the market structure. With price coupling first being introduced

\footnotetext{
$\dagger$ In the case of identical prices, a holiday might occur in one area but not the other. To avoid this, the holiday dummy returns a 1 if a holiday occurs in one of the two areas, and 0 otherwise.
} 
Model for the pair DE-FR hour 8

Equal price regime $\left(s_{t}=0\right)$

Non-equal price regime $\left(s_{t}=1\right)$

Area 1: DE

Area 2: FR

Conditional mean (optimal order $P=6, Q=0$ )

\begin{tabular}{lllllllll}
\hline$\hat{\phi}_{1}^{(0)}$ & 0.3458 & $(0.0381)$ & $\hat{\phi}_{1}^{(1)}$ & 0.4048 & $(0.0507)$ & $\hat{\phi}_{1}^{(2)}$ & 0.5036 & $(0.0481)$ \\
$\hat{\phi}_{2}^{(0)}$ & 0.1648 & $(0.0411)$ & $\hat{\phi}_{2}^{(1)}$ & 0.0810 & $(0.0508)$ & $\hat{\phi}_{2}^{(2)}$ & 0.1192 & $(0.0500)$ \\
$\hat{\phi}_{3}^{(0)}$ & 0.0134 & $(0.0396)$ & $\hat{\phi}_{3}^{(1)}$ & 0.0203 & $(0.0496)$ & $\hat{\phi}_{3}^{(2)}$ & 0.0611 & $(0.0502)$ \\
$\hat{\phi}_{4}^{(0)}$ & 0.0666 & $(0.0392)$ & $\hat{\phi}_{4}^{(1)}$ & 0.0329 & $(0.0483)$ & $\hat{\phi}_{4}^{(2)}$ & 0.0644 & $(0.0519)$ \\
$\hat{\phi}_{5}^{(0)}$ & 0.0420 & $(0.0377)$ & $\hat{\phi}_{5}^{(1)}$ & 0.0111 & $(0.0458)$ & $\hat{\phi}_{5}^{(2)}$ & 0.0043 & $(0.0491)$ \\
$\hat{\phi}_{6}^{(0)}$ & 0.0949 & $(0.0361)$ & $\hat{\phi}_{6}^{(1)}$ & 0.1910 & $(0.0431)$ & $\hat{\phi}_{6}^{(2)}$ & 0.1987 & $(0.0450)$
\end{tabular}

Conditional variance

\begin{tabular}{rllllllrr}
\hline$\hat{\omega}^{(0)}$ & 6.7896 & $(5.7732)$ & $\hat{\omega}^{(1)}$ & 8.2661 & $(8.1208)$ & $\hat{\omega}^{(2)}$ & 15.6209 & $(11.2384)$ \\
$\hat{\alpha}^{(0)}$ & 0.0487 & $(0.0410)$ & $\hat{\alpha}^{(1)}$ & 0.2058 & $(0.0880)$ & $\hat{\alpha}^{(2)}$ & 0.1218 & $(0.0787)$ \\
$\hat{\beta}^{(0)}$ & 0.7641 & $(0.1260)$ & $\hat{\beta}^{(1)}$ & 0.7359 & $(0.1681)$ & $\hat{\beta}^{(2)}$ & 0.7693 & $(0.1935)$
\end{tabular}

Marginal distribution (skew $t$ )

\begin{tabular}{lllllllrl}
\hline$\hat{\nu}^{(0)}$ & 4.8602 & $(1.0360)$ & $\hat{\nu}^{(1)}$ & 8.0736 & $(3.0836)$ & $\hat{\nu}^{(2)}$ & 11.5744 & $(4.9559)$ \\
$\hat{\lambda}^{(0)}$ & 0.0360 & $(0.0481)$ & $\hat{\lambda}^{(1)}$ & 0.0482 & $(0.0562)$ & $\hat{\lambda}^{(2)}$ & 0.0839 & $(0.0582)$ \\
\hline
\end{tabular}

Table 2: Parameter estimates for the DE-FR hour 8 model. Simulation based standard errors are given in parenthesis, and are based on 999 simulations following the procedure in Section 4.5.2.

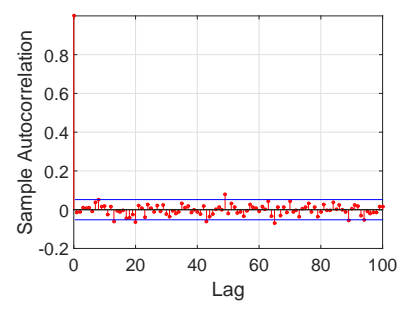

(a) DE - acf for std. residuals

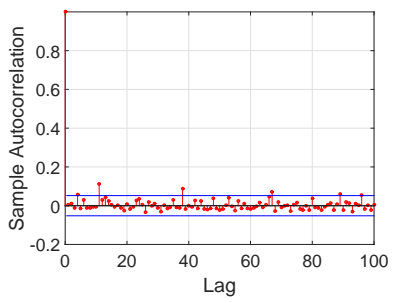

(b) DE - acf for squared std. residuals

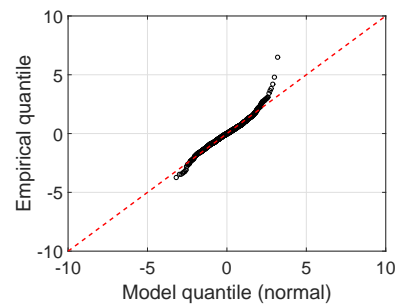

(e) DE - qq plot normal

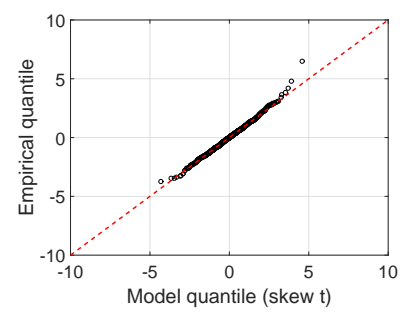

(f) DE - qq plot skew $t$

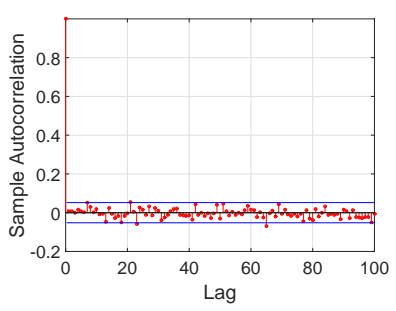

(c) FR - acf for std. residuals

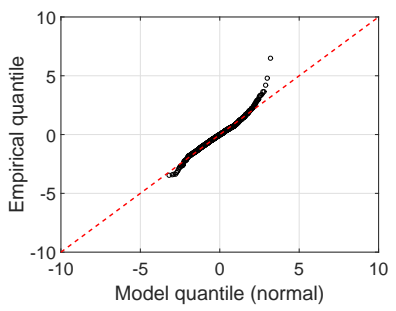

(g) FR - qq plot normal

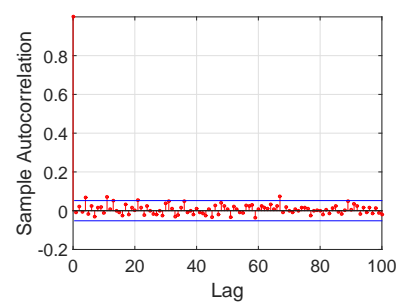

(d) FR - acf for squared std. residuals

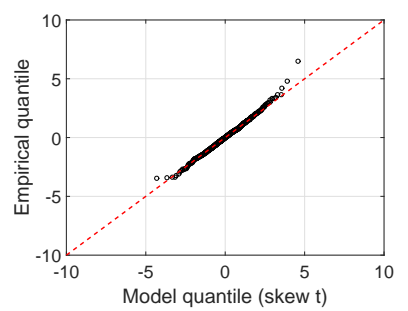

(h) FR - qq plot skew $t$

Figure 4: Sample autocorrelations and quantile plots of standardized residuals resulting from fitting regimeswitching AR-GARCH models to the DE-FR pair for hour 8. 
on 4 February 2014 as opposed to 10 November 2010, we wish to consider the pair DE-DK1 more closely. As mentioned earlier, the dynamics of the DE-DK1 spread has not changed visibly with the transition to price coupling, with equal prices having been observed prior as well as post price coupling. Therefore, we applied the same regime-switching AR-GARCH framework to this pair cf. Section 4.2. However, we wish to investigate whether a break in the dependence structure has occurred at the time of transitioning from volume coupling (VC) to price coupling (PC). If this were the case, the subsequent copula modeling would need to account for such a break.

We consider searching for a break in the rank correlation of the filtered prices (i.e., the standardized residuals obtained after applying the regime-switching AR-GARCH filter) corresponding to the non-equal price regime, at $t^{*}=4$ February 2014. Specifically, we wish to test

$$
H_{0}: \rho^{V C}=\rho^{P C} \quad \text { vs. } \quad H_{1}: \rho^{V C} \neq \rho^{P C},
$$

where $\rho^{V C}$ is the rank correlation (Spearman's $\rho$ ) of filtered prices corresponding to the non-equal price regime in the interval $\left[1, t^{*}\right]$, and $\rho^{P C}$ denotes the rank correlation (Spearman's $\rho$ ) in the interval $\left(t^{*}, T\right]$. A critical value for $\rho^{V C}-\rho^{P C}$ is obtained by using an i.i.d. bootstrap as in Patton (2013). ${ }^{\dagger}$

We perform the test for all 24 DE-DK1 models, and only find evidence against the null of equal rank correlation in 2 out of 24 cases (we consider a $5 \%$ significance level). Since we do not find stronger evidence for a break in the dependence, we decide to treat the pair DE-DK1 like all others in the subsequent analysis.

\subsection{Copula models}

Next, we turn to the study of the dependence between residual pairs obtained from the regime-switching ARGARCH models. As mentioned in Section 3, only the pairs of residuals belonging to the non-equal price regime are relevant in this context, and hence copula models are fitted to this data. We restrict our attention to five copulas, namely the Gaussian, Student $t$, Gumbel, Rotated Gumbel ${ }^{\ddagger}$ and Symmetrized Joe-Clayton (SJC) ${ }^{\S}$. The choice of these copulas is motivated by having models that can capture different types of dependence structures, and also by their extensive use in the economic and financial literature. In the interest of clarity, we summarize in Table 3 the key features of the copula models considered in this paper.

\begin{tabular}{lrrrr}
\hline & \multicolumn{4}{c}{ Properties of selected copulas } \\
Copula & Parameters & Asymmetric & Upper tail dep. & Lower tail dep. \\
\hline Gaussian & $\rho \in[-1,1]$ & No & 0 & 0 \\
Student $t$ & $\rho \in[-1,1], \nu>2$ & No & $2 t_{\nu+1}\left(-\sqrt{\nu+1} \sqrt{\frac{1-\rho}{1+\rho}}\right)$ & $2 t_{\nu+1}\left(-\sqrt{\nu+1} \sqrt{\frac{1-\rho}{1+\rho}}\right)$ \\
Gumbel & $\theta \geq 1$ & Yes & $2-2^{1 / \theta}$ & 0 \\
Rot. Gumbel & $\theta \geq 1$ & Yes & 0 & $2-2^{1 / \theta}$ \\
SJC & $\tau^{U} \in[0,1], \tau^{L} \in[0,1]$ & Yes/No & $\tau^{U}$ & $\tau^{L}$ \\
\hline
\end{tabular}

Table 3: Key features of the five selected copula models. In the tail dependence expression for the Student $t$ copula, $t_{\nu+1}$ refers to the probability density function of the Student $t$ distribution, with $\nu+1$ degrees of freedom. For further details on copulas and their properties, we refer to Joe (1997), Nelsen (1999) and Patton (2013).

The tail dependence terms appearing in Table 3 refer to measures of dependence between extreme events. For the case of positive dependence, the lower and upper tail dependence are defined as

$$
\begin{aligned}
\tau^{L} & =\lim _{q \rightarrow 0^{+}} \mathbb{P}\left(u_{t, 1} \leq q \mid u_{t, 2} \leq q\right)=\lim _{q \rightarrow 0^{+}} \frac{C(q, q)}{q} \\
\tau^{U} & =\lim _{q \rightarrow 1^{-}} \mathbb{P}\left(u_{t, 1}>q \mid u_{t, 2}>q\right)=\lim _{q \rightarrow 1^{-}} \frac{1-2 q+C(q, q)}{1-q},
\end{aligned}
$$

where $q$ denotes the quantile and $C$ denotes the bivariate copula distribution function. As it turns out, the expressions above have simple closed forms for many copulas. Specifically for the five copulas considered in this paper, tail dependence coefficients are easily computed using the expressions given in Table 3.

Turning to the estimation results, the five copula models are fitted to each residual pair; basing the model selection on the commonly used Akaike Information Criterion (AIC), we illustrate the preferred copula for each model in Table 4. The results reveal that the Gaussian copula is only chosen for 27 out of 96 models, confirming that linear correlation is, in most cases, not enough to describe the dependence between prices in

\footnotetext{
${ }^{\dagger}$ Note that by performing an i.i.d. bootstrap, we impose $H_{0}$.

$\ddagger$ A Rotated Gumbel (also called Survival Gumbel) is a Gumbel copula fitted to the rotated data (180 degrees rotation).

$\S$ For details regarding the SJC copula, we refer to Patton (2006).
} 
interconnected markets. The Student $t$ copula, which allows for symmetric upper and lower tail dependence, is preferred for 29 of the models. The even more flexible SJC copula, which allows for asymmetric upper and lower tail dependence (nesting the case of symmetric dependence), is chosen 26 times. Lastly, the Rotated Gumbel copula and the Gumbel copula, which allow for tail dependence in only one of the tails, are preferred in 8 and 6 cases, respectively.

\begin{tabular}{lrrrr}
\hline & & \multicolumn{2}{c}{ Optimal copula } & \\
Hour & DE-FR & DE-NL & NL-BE & DE-DK1 \\
\hline 0 & Student $t$ & SJC & Gaussian & Rot. Gum. \\
1 & Rot. Gum. & Gaussian & SJC & SJC \\
2 & SJC & Gaussian & Gaussian & SJC \\
3 & SJC & SJC & Gaussian & Student $t$ \\
4 & SJC & SJC & Gaussian & SJC \\
5 & SJC & SJC & Gaussian & Rot. Gum. \\
6 & Rot. Gum. & SJC & Student $t$ & SJC \\
7 & SJC & Student $t$ & Gaussian & Student $t$ \\
8 & Gumbel & SJC & SJC & Student $t$ \\
9 & SJC & Gaussian & Gaussian & Student $t$ \\
10 & Gumbel & SJC & Student $t$ & Student $t$ \\
11 & Gumbel & Gaussian & SJC & Student $t$ \\
12 & SJC & Gaussian & Student $t$ & Student $t$ \\
13 & Gumbel & Gaussian & Student $t$ & Student $t$ \\
14 & Student $t$ & Gaussian & SJC & Student $t$ \\
15 & Student $t$ & Gaussian & Gaussian & Student $t$ \\
16 & Student $t$ & Gaussian & Gaussian & Student $t$ \\
17 & Gumbel & SJC & Rot. Gum. & Gaussian \\
18 & Gumbel & Student $t$ & Student $t$ & Gaussian \\
19 & Student $t$ & Student $t$ & Gaussian & Gaussian \\
20 & Student $t$ & SJC & Student $t$ & Gaussian \\
21 & Rot. Gum. & Gaussian & SJC & Gaussian \\
22 & Student $t$ & Gaussian & Rot. Gum. & Rot. Gum. \\
23 & Student $t$ & Gaussian & Student $t$ & SJC \\
\hline
\end{tabular}

Table 4: Optimal copulas for each hourly model based on AIC.

\subsubsection{Tail dependence results}

To gain more insight regarding the optimal copulas listed in Table 4, we consider next their implied tail dependence. In Fig. 5, we plot the implied lower and upper tail dependence coefficients for all pairs of prices and all hours of the day. An i.i.d. bootstrap procedure was used to obtain the confidence intervals for the estimates (see e.g. Patton (2013) for more details). Moreover, the estimated lower and upper tail dependence coefficients grouped by peak (hours 8-19) and off-peak (hours 20-7) are reported in Table 5 .

\begin{tabular}{lcccr}
\hline & \multicolumn{4}{c}{ Lower tail dependence } \\
& DE-FR & DE-NL & NL-BE & DE-DK1 \\
\hline Mean peak & 0.0217 & 0.0324 & 0.0714 & 0.0338 \\
Mean off peak & 0.1830 & 0.1008 & 0.0776 & 0.2687 \\
\hline & DE-FR & Upper tail dependence & \\
& DE-NL & NL-BE & DE-DK1 \\
\hline Mean peak & 0.1899 & 0.0328 & 0.0576 & 0.0338 \\
Mean off peak & 0.0658 & 0.0261 & 0.0335 & 0.1170 \\
\hline
\end{tabular}

Table 5: Tail dependence implied by the optimal copulas listed in Table 4, grouped by peak and off-peak hours.

Recall that the analysis we perform in this section is based on filtered prices belonging to the non-equal price regime. Recall further that non-equal prices occur when there is a need to move more electricity across the border than what the available transmission capacity allows. Hence, we can interpret the lower (upper) tail dependence as being the probability of observing very low (high) prices in two interconnected markets conditional on the full utilization of the interconnector.

According to Fig. 5, the estimates of the preferred copulas translate into tail dependence coefficients that are often significantly different from zero - and this holds for selected hours in all price pairs. Further, a general tendency is that the lower tail dependence is stronger than the upper tail dependence during the off-peak hours (cf. Fig. 5 and Table 5). One explanation for this is that the probability of very low prices is higher during the off-peak hours, where the demand is lower. In a situation where two interconnected areas exhibit a low demand 


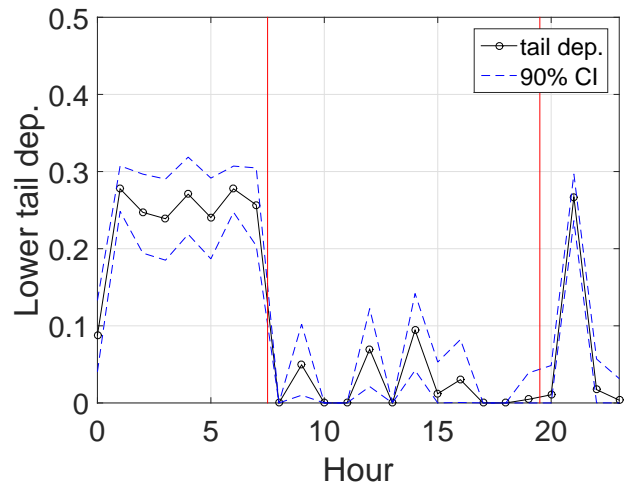

(a) DE and FR

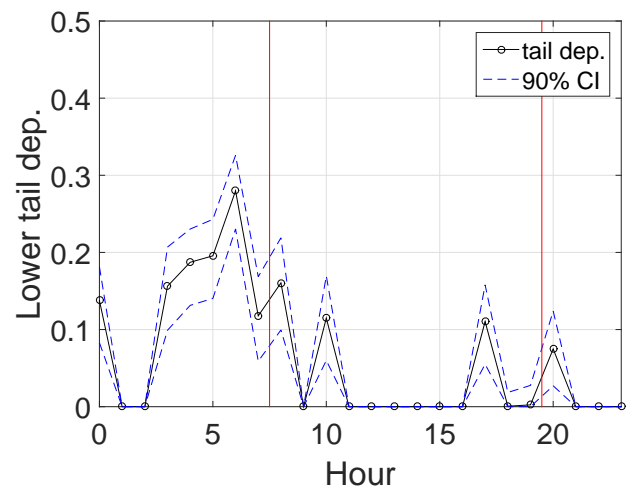

(c) DE and NL

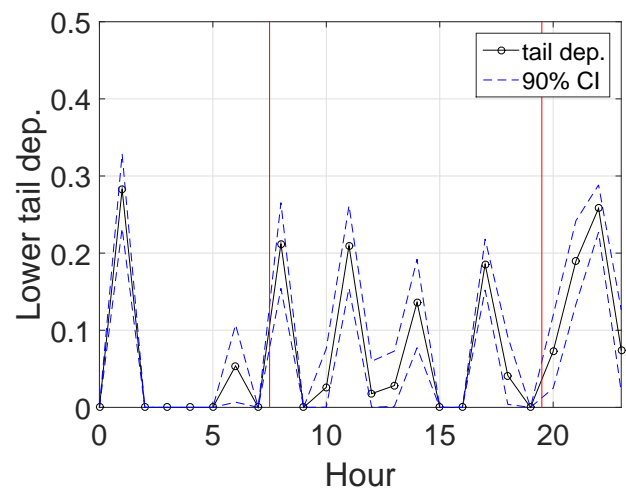

(e) NL and BE

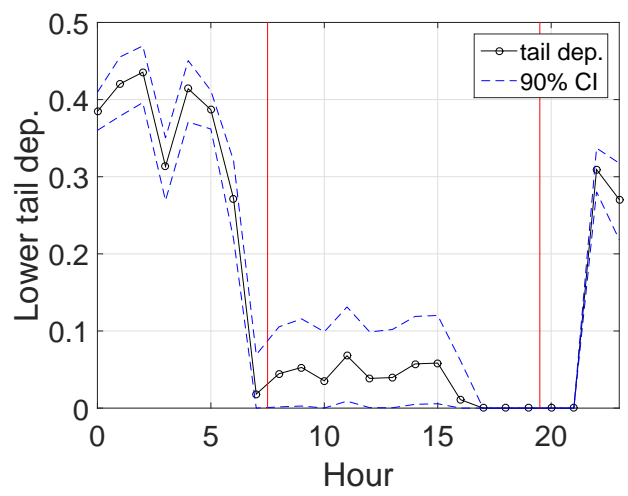

(g) DE and DK1

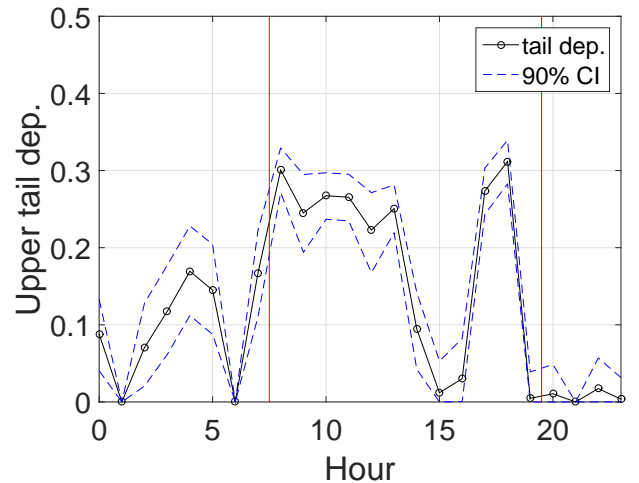

(b) DE and FR

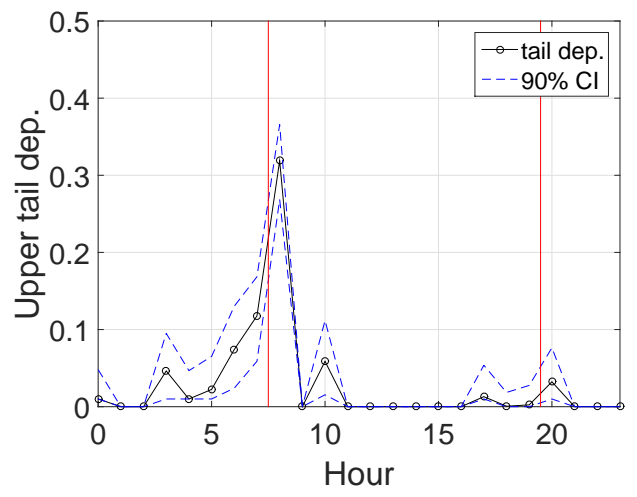

(d) DE and NL

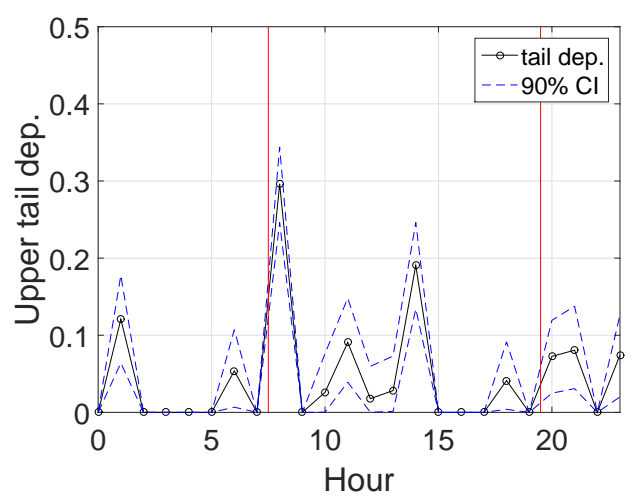

(f) NL and BE

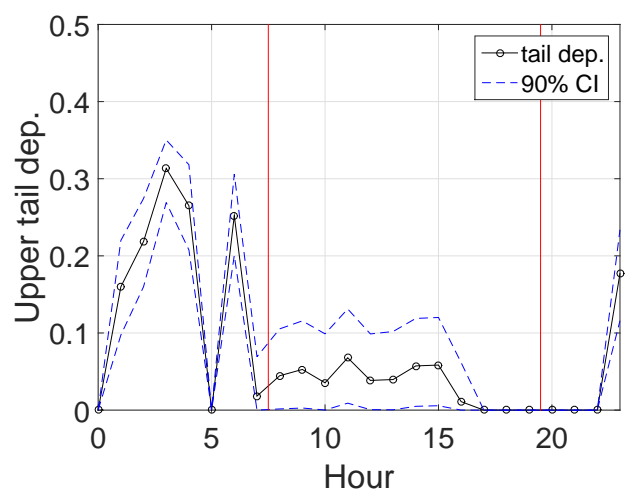

(h) DE and DK1

Figure 5: Estimated lower and upper tail dependence implied by the optimal copulas listed in Table 4. The tail dependence coefficients are obtained using the formulas given in Table 3. The red vertical lines indicate (from left to right) start and end of peak hours. 
and at the same time a high generation from renewable sources, e.g. wind energy generation, extremely low prices happening simultaneously is a likely outcome.

Another general feature we observe in Fig. 5 and Table 5 is that the lower tail dependence is usually higher during off-peak than during peak. Again here, we expect the lower demand during off-peak to be the main driver of this behavior. The most pronounced difference between peak and off-peak lower tail dependence is found for the DE-DK1 pair. This can be explained by the high wind infeed in both countries as well as similar weather conditions. Most wind turbines in Germany are placed in the northern part, that is, rather close to the Danish border, implying that high wind scenarios are likely to happen simultaneously in both countries.

While low prices happening simultaneously in two neighboring countries can be explained by e.g. low demand and/or high levels of renewables production, high prices occurring simultaneously can be explained by combinations of e.g. high demand, low renewables production, low temperatures, increasing marginal prices of the price setting unit (coal, gas), and plant outages. To provide an economic interpretation of particular events however, like the high upper tail dependence estimate for DE-NL for hour 8, can be very difficult, since a set of very specific combinations of events might have been the cause.

\subsection{Simulation}

Performing simulations from the proposed regime-switching AR-GARCH copula model, which is a central point in almost all applications, requires a last modeling element: A model for the probabilities of transitions between the two regimes.

\subsubsection{Transition matrices}

We let the regimes be determined by a Markov chain of order one, with transition probability matrix $\Pi$ :

$$
\Pi=\left[\begin{array}{cc}
\pi_{00} & 1-\pi_{00} \\
1-\pi_{11} & \pi_{11}
\end{array}\right] .
$$

The probability of prices being equal conditional on prices being equal at the previous time step is denoted by $\pi_{00}$. Similarly, $\pi_{11}$ denotes the probability of prices not being equal given that they were not equal at the previous time step. Recalling that the regimes are observable, the transition probabilities are simply estimated from the data as

$$
\begin{aligned}
& \hat{\pi}_{00}=\frac{N_{00}}{N_{00}+N_{01}}, \\
& \hat{\pi}_{11}=\frac{N_{11}}{N_{11}+N_{10}},
\end{aligned}
$$

where $N_{j k}$ denotes the number of times regime $j$ was followed by regime $k$, for $j=0,1$ and $k=0,1$.

\subsubsection{Simulation procedure}

Performing simulations from the proposed model is straightforward, but there are many steps involved. In order to create an overview, we briefly state the necessary steps below.

To generate a pair of (deseasonalized) prices $\left(y_{t, 1}, y_{t, 2}\right)$ at time $t$, we first generate the regime $s_{t}$ based on the transition probability matrix in Eq. (14).

If a) $s_{t}=0$, we are in the equal price regime, i.e., $y_{t, 1}=y_{t, 2}$, and a standardized residual $\eta_{t}$ is to be drawn from the univariate marginal distribution, which can be either $N(0,1)$ or Skew $t\left(0,1, \nu^{(0)}, \lambda^{(0)}\right)$ depending on the model specification. By applying Eqs. (2) and (1), respectively, a pair $\left(y_{t, 1}, y_{t, 2}\right)$ is subsequently obtained.

If $\mathbf{b}) s_{t}=1$, we have that $y_{t, 1} \neq y_{t, 2}$, and a pair $\left(u_{t, 1}, u_{t, 2}\right)$ is generated from the copula ${ }^{\dagger}$. A standardized residual pair $\left(\eta_{t, 1}, \eta_{t, 2}\right)$ is obtained by applying the inverse probability integral transform to the generated uniform pair $\left(u_{t, 1}, u_{t, 2}\right)$. This is done for each uniform individually, based on the univariate marginal distributions, i.e, $\mathrm{N}(0,1)$ or Skew $t\left(0,1, \nu^{(i)}, \lambda^{(i)},\right)$, for $i=1,2$. Finally, Eqs. (5) and (4) are employed, respectively, to generate a pair $\left(y_{t, 1}, y_{t, 2}\right)$.

\subsubsection{In-sample simulations}

To provide more evidence for the fit of the model, we perform in-sample simulations. Revisiting the example of DE-FR hour 8, we follow the procedure described in Section 4.5.2 to generate 1,000 sample paths of German and French prices of length $T-\max (P, Q)$, with $T$ denoting the sample size of the data, and $P$ and $Q$ referring to the order of the model for the conditional mean, cf. Eqs. (1) and (4). $\ddagger$ In Fig. 6, we show quantile plots of

\footnotetext{
${ }^{\dagger}$ Generating random draws from a copula is based on the inverse of the conditional copula functions (often referred to as $h$-functions). See e.g. Aas et al. (2009) for more details.

$\ddagger$ To start the simulation, we use the first $\max (P, Q)$ prices. Regarding $\hat{\sigma}_{0, i}^{2}$, for $i=1,2$, we use the sample variance of the data.
} 
actual prices vs. simulated prices, and quantile plots of actual non-zero spreads vs. simulated non-zero spreads, with the results revealing a satisfactory in-sample fit.

Similar in-sample fits were obtained for the other pairs regarding quantile plots of the individual price series. For the spreads on the other hand, the model does not always perform very well when considering matching both tails. For some hours of the day regarding DE-NL, the model assigns slightly more mass to the right tail of the distribution, while being able to capture the left tail nicely. As can be seen in Fig. 2 and Table 1, cases where the Dutch price is above the German price are either extremely few or none at all, explaining perhaps the inability of the model to provide a sufficient explanation of the right tail.

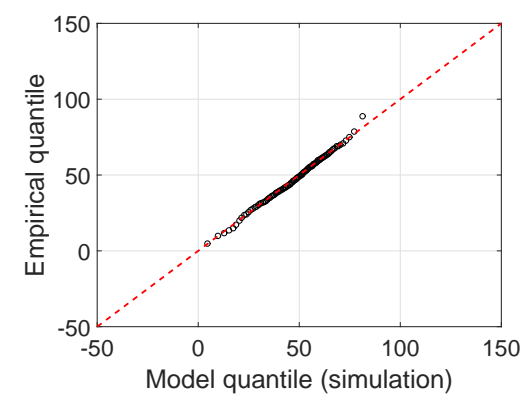

(a) DE price

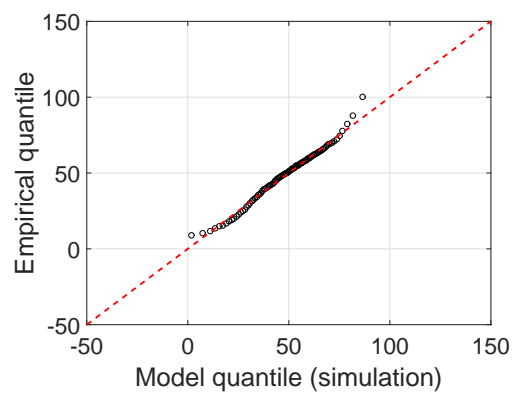

(b) FR price

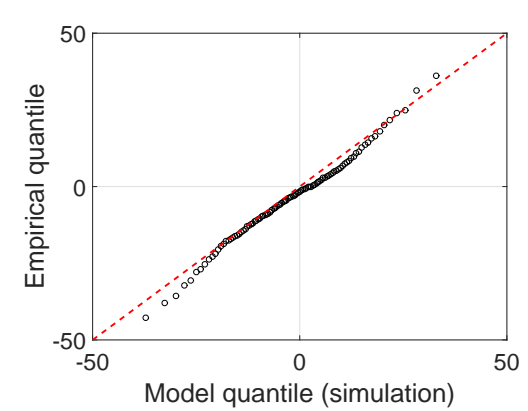

(c) DE-FR spread

Figure 6: Quantile plots of price and (non-zero) spread data vs. simulated prices and (non-zero) spreads from the fitted model with skew $t$ margins and a Gumbel copula cf. Table 4.

\section{$5 \quad$ Financial transmission rights}

The presence of heavy tails in the individual price distributions, asymmetric dependence and tail dependence between prices can have significant implications when considering derivatives whose payoffs depend on the price spread. To investigate such effects, we consider in this section the pricing of financial transmission rights as a first application.

A financial transmission right (FTR) is a spread option written on the hourly day-ahead electricity price difference in two interconnected areas. It gives the option holder the right to "transfer electricity across the border" if the price difference is positive for the corresponding hour. In reality, no physical cross-border transfer of electricity is undertaken as the rights are settled financially, hence the name financial transmission right.

A particular feature of such rights is that delivery periods are limited to an entire year or month. When entering e.g. a monthly FTR, the option holder actually enters a string of hourly spread options, and has the right to exercise at each hour in that month. Also interesting is that FTRs are linked to the actual available capacity of the interconnector, and they are offered by the transmission system operators (TSO) through explicit auctions.

For the markets we consider here, either FTRs or physical transmission rights (PTRs) with a Use-it-or-Sell-it (UIOSI) feature are available. For the latter type, the option holder has the right to either undertake physical cross-border delivery, or simply sell the option back to the market and receive the price difference whenever this is positive. Thus, PTRs with UIOSI can function as purely financial instruments, and have the same payoff structure as the FTRs. Since we are only interested in the financial aspect of transmission rights, we shall use the term FTR regardless of whether an FTR can in reality be traded for a specific pair of prices. ${ }^{\dagger}$

Let us denote by $V\left(t_{0}, T\right)$ the value of a financial transmission right at time $t_{0}$, with delivery at $T . V\left(t_{0}, T\right)$ can be obtained by the usual practice of computing the discounted value of the expected payoff, where the expectation is taken under a pricing measure $\mathbb{Q}$ (see e.g. Benth et al. (2008)):

$$
V\left(t_{0}, T\right)=\mathrm{e}^{r\left(T-t_{0}\right)} \mathbb{E}_{t_{0}}^{\mathbb{Q}}\left[\max \left(P_{T}^{1}-P_{T}^{2}, 0\right)\right]
$$

In Eq. (15), $P_{T}^{i}$ denotes the hourly day-ahead electricity price corresponding to the time period $T$ in area $i$, where $i=1,2$. Furthermore, $r$ denotes the risk-free rate, which we for simplicity set to zero in the following analysis.

Since delivery periods for FTRs are limited to a month or a year, the value of e.g. a monthly FTR is simply obtained as the sum of all individual hourly spread option values in that month, which in turn are obtained

\footnotetext{
${ }^{\dagger}$ For a detailed description of the different types of transmission rights, also before and after the coupling of CWE, we refer to Füss et al. (2015).
} 
using Eq. (15). Thus, the holder of a monthly FTR will have the right to exchange electricity in area 2 for electricity in area 1, if the price difference is positive, during each hour of that month.

Having established a modeling framework for pairs of day-ahead electricity prices in interconnected areas, FTR prices can be obtained by Monte Carlo simulation, following the simulation procedure described in Section 4.5.2. We note however that computing expectations based on simulations from a model that has been fitted to historical data would yield an option price under the objective measure $\mathbb{P}$, and not the pricing measure $\mathbb{Q}$. We will make the simplifying assumption of $\mathbb{P}=\mathbb{Q}$, arguing that a change of measure would not alter the overall conclusions regarding the impacts of heavy tails and choice of copula on option prices. For further discussions regarding measure change in the context of electricity markets we refer to Burger et al. (2004), Benth and Meyer-Brandis (2009), and Benth and Schmeck (2014).

To investigate the effects of heavy tails and choice of copula model, we consider here the pricing of FTRs for the months June and December 2015 as examples. The valuation date is set to $t_{0}=20$ May 2015, which corresponds to the end of our sample. Since we model each hour of the day separately, we will limit ourselves to pricing monthly FTRs with delivery during one single hour of the day, for all days in June and December. This way, we avoid averaging effects to some extent and showcase the different behavior among the hours of the day. Specifically, we consider hour 2, 8, 14 and 20, for all pairs of prices, and flows in both directions. The pricing formula for e.g. the flow direction $\mathrm{DE} \rightarrow \mathrm{FR}$ during each hour 2 in June is given by

$$
V^{\mathrm{DE} \rightarrow \mathrm{FR}}\left(t_{0}, T_{1}, T_{2}, h=2\right)=\sum_{t=T_{1}}^{T_{2}} \mathbb{E}_{t_{0}}^{\mathbb{P}}\left[\max \left(P_{t}^{F R}-P_{t}^{D E}, 0\right)\right],
$$

where $t_{0}=20$ May 2015, $T_{1}=1$ June 2015, and $T_{2}=30$ June 2015. $P_{t}^{F R}$ and $P_{t}^{D E}$ denote the hour 2 prices in France and Germany, respectively, for a given day $t \in\left[T_{1}, T_{2}\right]$.

We perform option price calculations using three different models: First, a model with normal margins and a Gaussian copula (Model 1); second, a model with skew $t$ margins and a Gaussian copula (Model 2), and third, a model with skew $t$ margins and the preferred copula cf. Table 4 (Model 3). By construction, Model 1 is a naive model since it imposes no heavy tails, no asymmetry and no tail dependence. Model 2 on the other hand allows for heavy tails but imposes symmetry and no tail dependence, while Model 3 allows for heavy tails, and can allow for tail dependence and asymmetry (note that preferred copulas are in some cases the Gaussian copula according to Table 4). In the interest of clarity, we summarize the characteristics of the above-mentioned models in Table 6.

\begin{tabular}{lrrr}
\hline & $\begin{array}{r}\text { Model 1 } \\
\text { Normal margins \& } \\
\text { Gaussian copula }\end{array}$ & $\begin{array}{r}\text { Model 2 } \\
\text { Skew } t \text { margins \& } \\
\text { Gaussian copula }\end{array}$ & $\begin{array}{r}\text { Model 3 } \\
\text { optimal copula }\end{array}$ \\
\cline { 2 - 4 } Heavy tails (margins) & $\times$ & $\checkmark$ & $\checkmark$ \\
Tail dependence & $\times$ & $\times$ & $\times$ or $\checkmark$ \\
Asymmetric dependence & $\times$ & $\times$ & or $\checkmark$ \\
\hline
\end{tabular}

Table 6: Model characteristics for the three different models used to compute FTR prices. The optimal copula entering Model 3 refers to the preferred specification cf. Table 4; this model may or may not allow for tail dependence and asymmetric dependence.

The pricing results are displayed in Table 7, and are based on 200,000 Monte Carlo simulations from the fitted regime-switching AR-GARCH copula models from Section 4. Price 1, 2 and 3 in Table 7 refer to prices obtained from Model 1, 2 and 3, respectively, and are given in EUR/MWh. $\Delta_{1}$ refers to the relative difference between Price 1 and 2, measuring the effect of introducing heavy tails in the model. $\Delta_{2}$ refers to the relative difference between Price 2 and 3, measuring the effect of using a better fitting copula, which in contrast to the Gaussian copula, allows for tail dependence and in some cases asymmetry. Notice that if the Gaussian copula was chosen as the optimal copula cf. Table 4, no price for the corresponding option was computed with Model 3 , since Model 3 then corresponds to Model 2.

Regarding the robustness of option price estimates, we note that the same option prices were estimated based on 100,000 and 200,000 Monte Carlo simulations, yielding estimates that are generally accurate to two or three decimal places. Furthermore, the same random number generator was used to compute option prices across competing models, thus allowing us to solely concentrate on the effects induced by the different marginal distributions and copulas.

According to Table 7, introducing heavy tails can have a significant effect in terms of magnitude, with the maximum reduction in the option price being as high as $-45 \%$, and the maximum increase reaching $+28 \%$. In terms of sign, the effects are mixed, which might be surprising at first. In the case of e.g. an out-of-the-money option written on one underlying asset, heavy tails would imply an increase in the option price, since more mass is assigned to extreme events. In the case of spread options however, where the payoff depends on the price 


\begin{tabular}{|c|c|c|c|c|c|c|c|c|c|c|}
\hline & \multicolumn{10}{|c|}{ Delivery during June } \\
\hline & Price 1 & Price 2 & Price 3 & $\Delta_{1}$ & $\Delta_{2}$ & Price 1 & Price 2 & Price 3 & $\Delta_{1}$ & $\Delta_{2}$ \\
\hline & \multicolumn{5}{|c|}{$\mathbf{F R} \rightarrow \mathbf{D E}$} & \multicolumn{5}{|c|}{ DE $\rightarrow$ FR } \\
\hline Hour 2 & 3.0621 & 2.8952 & 2.8714 & $-5.45 \%$ & $-0.82 \%$ & 1.9482 & 1.7830 & 1.7608 & $-8.48 \%$ & $-1.25 \%$ \\
\hline Hour 8 & 2.5707 & 2.5518 & 2.5298 & $-0.74 \%$ & $-0.86 \%$ & 1.3009 & 1.2818 & 1.2657 & $-1.47 \%$ & $-1.26 \%$ \\
\hline Hour 14 & 0.7294 & 0.6528 & 0.6365 & $-10.50 \%$ & $-2.50 \%$ & 5.9410 & 5.8640 & 5.8461 & $-1.30 \%$ & $-0.31 \%$ \\
\hline Hour 20 & 3.7612 & 3.7539 & 3.7451 & $-0.19 \%$ & $-0.23 \%$ & 0.9362 & 0.9282 & 0.9191 & $-0.85 \%$ & $-0.98 \%$ \\
\hline & \multicolumn{5}{|c|}{$\mathbf{N L} \rightarrow \mathrm{DE}$} & \multicolumn{5}{|c|}{$\mathrm{DE} \rightarrow \mathrm{NL}$} \\
\hline Hour 2 & 0.7644 & 0.4850 & - & $-36.55 \%$ & - & 6.8804 & 6.6040 & - & $-4.02 \%$ & - \\
\hline Hour 8 & 0.1948 & 0.2106 & 0.1939 & $8.11 \%$ & $-7.93 \%$ & 5.1074 & 5.1223 & 5.1198 & $0.29 \%$ & $-0.05 \%$ \\
\hline Hour 14 & 0.1297 & 0.0898 & - & $-30.76 \%$ & - & 12.4058 & 12.3658 & - & $-0.32 \%$ & - \\
\hline \multirow[t]{2}{*}{ Hour 20} & 0.2574 & 0.2616 & 0.2677 & $1.63 \%$ & $2.33 \%$ & 6.0492 & 6.0517 & 6.0680 & $0.04 \%$ & $0.27 \%$ \\
\hline & \multicolumn{5}{|c|}{$\mathrm{BE} \rightarrow \mathrm{NL}$} & \multicolumn{5}{|c|}{$\mathrm{NL} \rightarrow \mathrm{BE}$} \\
\hline Hour 2 & 2.0140 & 1.9936 & - & $-1.01 \%$ & - & 0.8381 & 0.8169 & - & $-2.53 \%$ & - \\
\hline Hour 8 & 1.1731 & 1.1488 & 1.1253 & $-2.07 \%$ & $-2.05 \%$ & 1.5565 & 1.5307 & 1.5103 & $-1.66 \%$ & $-1.33 \%$ \\
\hline Hour 14 & 4.3010 & 4.3039 & 4.2978 & $0.07 \%$ & $-0.14 \%$ & 0.2761 & 0.2776 & 0.2696 & $0.54 \%$ & $-2.88 \%$ \\
\hline \multirow[t]{2}{*}{ Hour 20} & 2.7042 & 2.6650 & 2.6495 & $-1.45 \%$ & $-0.58 \%$ & 0.8726 & 0.8322 & 0.8163 & $-4.63 \%$ & $-1.91 \%$ \\
\hline & \multicolumn{5}{|c|}{$\mathrm{DK} 1 \rightarrow \mathrm{DE}$} & \multicolumn{5}{|c|}{$\mathrm{DE} \rightarrow \mathrm{DK} 1$} \\
\hline Hour 2 & 1.5204 & 1.3093 & 1.2060 & $-13.88 \%$ & $-7.89 \%$ & 3.9435 & 3.7318 & 3.6144 & $-5.37 \%$ & $-3.15 \%$ \\
\hline Hour 8 & 4.4038 & 4.3768 & 4.3417 & $-0.61 \%$ & $-0.80 \%$ & 2.5883 & 2.5616 & 2.5260 & $-1.03 \%$ & $-1.39 \%$ \\
\hline Hour 14 & 1.2316 & 1.1128 & 1.0772 & $-9.65 \%$ & $-3.20 \%$ & 5.7916 & 5.6728 & 5.6374 & $-2.05 \%$ & $-0.62 \%$ \\
\hline Hour 20 & 6.0021 & 6.0090 & - & $0.11 \%$ & - & 0.9293 & 0.9357 & - & $0.69 \%$ & - \\
\hline
\end{tabular}

\begin{tabular}{|c|c|c|c|c|c|c|c|c|c|c|}
\hline & \multicolumn{10}{|c|}{ Delivery during December } \\
\hline & Price 1 & Price 2 & Price 3 & $\Delta_{1}$ & $\Delta_{2}$ & Price 1 & Price 2 & Price 3 & $\Delta_{1}$ & $\Delta_{2}$ \\
\hline & \multicolumn{5}{|c|}{$\mathbf{F R} \rightarrow \mathbf{D E}$} & \multicolumn{5}{|c|}{$\mathrm{DE} \rightarrow \mathrm{FR}$} \\
\hline Hour 2 & 0.1771 & 0.1155 & 0.1113 & $-34.78 \%$ & $-3.64 \%$ & 11.0058 & 10.9432 & 10.9545 & $-0.57 \%$ & $0.10 \%$ \\
\hline Hour 8 & 0.1885 & 0.1909 & 0.1933 & $1.27 \%$ & $1.26 \%$ & 6.9024 & 6.9054 & 6.8953 & $0.04 \%$ & $-0.15 \%$ \\
\hline Hour 14 & 0.4627 & 0.4193 & 0.4139 & $-9.38 \%$ & $-1.29 \%$ & 7.6324 & 7.5900 & 7.5841 & $-0.56 \%$ & $-0.08 \%$ \\
\hline \multirow[t]{2}{*}{ Hour 20} & 0.1303 & 0.1363 & 0.1332 & $4.60 \%$ & $-2.27 \%$ & 9.1461 & 9.1526 & 9.1494 & $0.07 \%$ & $-0.03 \%$ \\
\hline & \multicolumn{5}{|c|}{$\mathbf{N L} \rightarrow \mathbf{D E}$} & \multicolumn{5}{|c|}{$\mathrm{DE} \rightarrow \mathrm{NL}$} \\
\hline Hour 2 & 0.3427 & 0.1868 & - & $-45.49 \%$ & - & 9.8521 & 9.6975 & - & $-1.57 \%$ & - \\
\hline Hour 8 & 0.0986 & 0.1261 & 0.1092 & $27.89 \%$ & $-13.40 \%$ & 6.4267 & 6.4546 & 6.4380 & $0.43 \%$ & $-0.26 \%$ \\
\hline Hour 14 & 0.2723 & 0.2016 & - & $-25.96 \%$ & - & 10.3227 & 10.2520 & - & $-0.68 \%$ & - \\
\hline \multirow[t]{2}{*}{ Hour 20} & 0.1058 & 0.1145 & 0.1178 & $8.22 \%$ & $2.88 \%$ & 8.0795 & 8.0894 & 8.0925 & $0.12 \%$ & $0.04 \%$ \\
\hline & \multicolumn{5}{|c|}{$\mathrm{BE} \rightarrow \mathrm{NL}$} & \multicolumn{5}{|c|}{$\mathrm{NL} \rightarrow \mathrm{BE}$} \\
\hline Hour 2 & 0.7064 & 0.7091 & - & $0.38 \%$ & - & 2.3100 & 2.3129 & - & $0.13 \%$ & - \\
\hline Hour 8 & 0.6977 & 0.6845 & 0.6644 & $-1.89 \%$ & $-2.94 \%$ & 2.4609 & 2.4479 & 2.4278 & $-0.53 \%$ & $-0.82 \%$ \\
\hline Hour 14 & 1.8068 & 1.7556 & 1.7470 & $-2.83 \%$ & $-0.49 \%$ & 1.3769 & 1.3271 & 1.3136 & $-3.62 \%$ & $-1.02 \%$ \\
\hline \multirow[t]{2}{*}{ Hour 20} & 1.1500 & 1.1291 & 1.1186 & $-1.82 \%$ & $-0.93 \%$ & 2.5007 & 2.4806 & 2.4697 & $-0.80 \%$ & $-0.44 \%$ \\
\hline & \multicolumn{5}{|c|}{ DK1 $\rightarrow$ DE } & \multicolumn{5}{|c|}{$\mathrm{DE} \rightarrow \mathrm{DK} 1$} \\
\hline Hour 2 & 1.3579 & 1.1409 & 1.0204 & $-15.98 \%$ & $-10.56 \%$ & 4.6786 & 4.4630 & 4.3492 & $-4.61 \%$ & $-2.53 \%$ \\
\hline Hour 8 & 7.0992 & 7.1558 & 7.1327 & $0.80 \%$ & $-0.32 \%$ & 1.4306 & 1.4904 & 1.4680 & $4.18 \%$ & $-1.66 \%$ \\
\hline Hour 14 & 4.0953 & 4.0324 & 3.9957 & $-1.54 \%$ & $-0.91 \%$ & 2.3401 & 2.2786 & 2.2422 & $-2.63 \%$ & $-1.29 \%$ \\
\hline Hour 20 & 8.4618 & 8.5114 & - & $0.59 \%$ & - & 0.5290 & 0.5807 & - & $9.77 \%$ & - \\
\hline
\end{tabular}

Table 7: Option prices in EUR/MWh with delivery in June and December 2015, during the specified hour. Price 1 refers to an option price obtained from models with normal margins and a Gaussian copula. Price 2 refers to models with skew $t$ margins and a Gaussian copula, and Price 3 refers to models with skew $t$ margins and the optimal copula cf. Table 4. $\Delta_{1}$ refers to the difference between Price 1 and 2 , while $\Delta_{2}$ refers to the difference between Price 2 and 3. 


\begin{tabular}{lrrr}
\hline & Kendall's $\tau_{1}$ & Kendall's $\tau_{2}$ & Kendall's $\tau_{3}$ \\
\hline & \multicolumn{3}{c}{ DE and FR } \\
Hour 2 & 0.2454 & 0.2315 & 0.2116 \\
Hour 8 & 0.2446 & 0.2434 & 0.2350 \\
Hour 14 & 0.1961 & 0.2093 & 0.2126 \\
Hour 20 & 0.2113 & 0.2124 & 0.2132 \\
& & DE and NL & \\
Hour 2 & 0.1802 & 0.1917 & - \\
Hour 8 & 0.3149 & 0.2961 & 0.2742 \\
Hour 14 & 0.1279 & 0.1499 & - \\
Hour 20 & 0.1445 & 0.1397 & 0.1242 \\
& & NL and BE & \\
Hour 2 & 0.2658 & 0.2706 & - \\
Hour 8 & 0.3035 & 0.2951 & 0.2812 \\
Hour 14 & 0.2398 & 0.2277 & 0.2137 \\
Hour 20 & 0.1930 & 0.1929 & 0.1941 \\
& & DE and DK1 & \\
Hour 2 & 0.3984 & 0.3545 & 0.3452 \\
Hour 8 & 0.3243 & 0.3070 & 0.3099 \\
Hour 14 & 0.2883 & 0.3071 & 0.3130 \\
Hour 20 & 0.2546 & 0.2482 & - \\
\hline
\end{tabular}

Table 8: Kendall's $\tau$ implied by the three models specified in Table 6, for the same pairs of prices and hours of the day as in Table 7.

differential, heavy tails might or might not cancel out, leading to both decreases and increases. Another factor influencing both the sign and the magnitude is the dependence implied by the Gaussian copula, which is not exactly the same for the models with normal margins as for the those with skew $t$ margins. To illustrate this variation, we compute the Kendall's $\tau$ implied by the models specified in Table 6 . The results are reported in Table 8, revealing differences which naturally impact the option prices.

Also noticeable in the context of heavy tails is that when comparing the same option in the two different flow directions, the effect is always magnified for the direction that is least in-the-money. The more difference there is between the moneyness of the options, the more difference there is in the effect of heavy tails. This is of course expected, since out-of-the-money options are more sensitive to changes in the tail behavior of a distribution.

When considering the effects of using a better fitting copula, the results generally imply a reduction in the option prices, with a maximum drop amounting to $-13 \%$. As with heavy tails, the effects are magnified for out-of-the-money options. These findings are expected if considering the isolated effect of tail dependence: Allowing prices to move together in extreme events lowers the probability of obtaining extreme price differences in the simulated future payoffs, which ultimately lowers the option price. Furthermore, the moneyness of the option plays an important role in this context, since it decides the importance of the joint tail behavior for the option price.

Although tail dependence is included in the effect measured by $\Delta_{2}$, we stress that tail dependence is not the only influencing factor, and hence its impact cannot be separated and measured explicitly. Equally important is that $\Delta_{2}$ can include the effects of asymmetry, and simply measures the impact of assuming a dependence structure that is different from the Gaussian one. To emphasize this point, we again refer to Kendall's $\tau$ computed in Table 8, which makes the results comparable across copula models. According to this measure, some differences are observed in the dependence implied by Models 2 and 3. With everything else being equal, a reduction in Kendall's $\tau$ implies an increase in the option price, which would counteract the effects of tail dependence. Similarly, an increase in Kendall's $\tau$ implies a decrease in the option price, possibly adding to the reduction implied by tail dependence.

Overall, heavy tails and a better fitting copula are most important to consider in the context of out-of-themoney options - and as we can see from Table 7, there are many such options for the interconnected electricity markets we analyze here.

Lastly, we note that the seasonal behavior of prices and the electricity flow patterns discussed in Section 2 are of course reflected in the option prices. For the pair DE-FR for example, we observe that the direction FR $\rightarrow$ DE is more in-the-money than $\mathrm{DE} \rightarrow \mathrm{FR}$ for the summer month June, during hours 2, 8 and 20. During hour 14, when solar production in Germany is at its peak, the situation reverses. During the winter month December, $\mathrm{FR} \rightarrow \mathrm{DE}$ is deep out-of-the-money compared to $\mathrm{DE} \rightarrow \mathrm{FR}$, which is due to increased demand and a high wind power production in Germany. Also for the pair DE-NL, the fact that Netherlands is a significant importer of German power is reflected in the option prices, with $\mathrm{NL} \rightarrow \mathrm{DE}$ being very cheap compared to DE $\rightarrow \mathrm{NL}$. 


\section{$6 \quad$ Forecasting of tail quantiles}

To provide more empirical evidence for the proposed modeling framework, we consider in this section the forecasting of tail quantiles as a second application. This can be of interest to parties with physical or financial positions in two interconnected markets, portfolio managers, and also risk managers who wish to asses worst-case scenarios.

Clearly, the proposed regime-switching AR-GARCH copula model is a sophisticated approach to forecasting the tail quantiles of the price spread distributions. Therefore, aside from wishing to illustrate the effects of heavy tails and a copula that allows for asymmetry and tail dependence, we also find it relevant to compare the performance of the proposed copula framework with a much simpler approach. We thus perform the forecasting exercise with the following three models:

- First, we consider the regime-switching AR-GARCH model in Eqs. (1) - (6) with skew $t$ margins and a SJC copula. This model will be referred to as Skew $t /$ SJC.

- Second, we consider the regime-switching AR-GARCH model in Eqs. (1) - (6) with normal margins and a Gaussian copula. This model will be referred to as N/Gaussian.

- Third, we consider a naive approach that is very different from the copula framework. Specifically, we apply a regime-switching $\mathrm{AR}(1)$ model to the deseasonalized spread $z$ :

$$
z_{t}=\phi_{s_{t}} z_{t-1}+\epsilon_{t, s_{t}}, \quad \epsilon_{t} \sim \mathrm{N}\left(0, \sigma_{s_{t}}^{2}\right) .
$$

To correct the spread time series for deterministic seasonality, we apply the seasonal function in Eq. (13). The regimes in this univariate model are the same as before: Whenever $s_{t}=0$, we are in the zero spread (equal prices) regime; if $s_{t}=1$, we are in the non-zero spread (non-equal prices) regime. Further, we again let the regimes be determined by a Markov chain of order one. As far as the regimes are concerned, the only difference from the copula approach is that whenever $s_{t}=0$ in the univariate spread model, all the model parameters are zero. This model will be referred to as Univariate.

To evaluate the forecasting power of each model, we perform out-of-sample day-ahead forecasts of the tail quantiles for the pair DE-DK1 at hours $0,1, \ldots, 6$. This choice is motivated by the high degree of asymmetry and tail dependence obtained for this pair during most off-peak hours, cf. Fig. 5. On one hand, forecasting with the Skew $t$ /SJC and the N/Gaussian models enable us to investigate the effects of heavy tails and those of using a copula that allows for asymmetry and tail dependence. On the other hand, employing a very different and much simpler model (the Univariate model) reveals whether or not the copula modeling framework is at all advantageous in this context.

Specifically, we consider the $5 \%, 1 \%$ and $0.5 \%$ quantiles of the spread distributions for a period of two years (730 days), corresponding to 21 May 2013 - 20 May 2015. We employ an "expanding window" estimation, where an out-of-sample forecast for period $t+s$ is obtained by fitting the models to data in the interval $[1, t+s-1]$. The models are re-estimated at each step as we progress through the out-of-sample period, and each forecast is based on 100,000 Monte Carlo simulations.

The coverage results for the forecasts are summarized in Table 9 , where both tails of the spread distributions are considered, i.e. the $5 \%, 1 \%$ and $0.5 \%$ quantile forecasts for the distribution of both DE-DK1 and DK1-DE prices. To provide a visual example, we also plot in Fig. 7 the results obtained for hour 0 with the Skew $t /$ SJC model.

While the coverage results in Table 9 are informative, they are not sufficient as to draw conclusions on model adequacy. To asses the performance of the models, we hence conduct formal statistical testing. We consider the three likelihood ratio tests proposed in Christoffersen (1998), namely the test of correct unconditional coverage $\left(\mathrm{LR}_{\mathrm{uc}}\right)$, independence $\left(\mathrm{LR}_{\mathrm{ind}}\right)$, and correct conditional coverage $\left(\mathrm{LR}_{\mathrm{cc}}\right)$. While $\mathrm{LR}_{\mathrm{uc}}$ and $\mathrm{LR}_{\text {ind }}$ are concerned with exceedance proportions and the order of exceedance occurrences, respectively, $L_{\mathrm{cc}}$ is a complete test that enables the joint testing of coverage and independence. When conditioning on the fist observation - as we do here - Christoffersen (1998) shows that $\mathrm{LR}_{\mathrm{cc}}=\mathrm{LR}_{\mathrm{uc}}+\mathrm{LR}_{\text {ind }} .^{\dagger}$

For the $5 \%$ out-of-sample quantile forecasts, we report in Table 9 the $p$-values obtained by performing all three tests mentioned above. When no concecutive exceedances are present, we note that the independence test and hence the conditional coverage test are not defined. In such situations, the $p$-value is set to NaN. Since the case of no consecutive exceedances is extremely common for the $1 \%$ and $0.5 \%$ quantile forecasts, we confine ourselves to reporting the results corresponding to the test of unconditional coverage for these quantiles.

Generally, the $5 \%$ out-of-sample quantile forecasts pass the independence test across all models. We only encounter two cases where independence is rejected: Once for the N/Gaussian model, and once for the Univariate

\footnotetext{
${ }^{\dagger}$ For technical details and a thorough description of all three tests we refer the interested reader to Christoffersen (1998).
} 


\begin{tabular}{|c|c|c|c|c|c|c|c|c|}
\hline & \multicolumn{6}{|c|}{ DK1-DE } & & \\
\hline & \multicolumn{4}{|c|}{$q=0.05(36.5)$} & \multicolumn{2}{|c|}{$q=0.01(7.3)$} & \multicolumn{2}{|c|}{$q=0.005(3.65)$} \\
\hline \multicolumn{9}{|c|}{ Hour 0} \\
\hline Skew $t /$ SJC & $0.0548(40)$ & 0.5523 & 0.2416 & 0.4222 & $0.0110(8)$ & 0.7947 & $0.0082(6)$ & 0.2582 \\
\hline N/Gaussian & $0.0466(34)$ & 0.6738 & 0.7393 & 0.8659 & $0.0123(9)$ & 0.5392 & $0.0096(7)$ & 0.1182 \\
\hline Univariate & $0.0274(20)$ & 0.0023 & 0.5721 & 0.0082 & $0.0055(4)$ & 0.1805 & $0.0055(4)$ & 0.8544 \\
\hline \multicolumn{9}{|c|}{ Hour 1} \\
\hline Skew $t /$ SJC & $0.0644(47)$ & 0.0855 & 0.5678 & 0.1937 & $0.0178(13)$ & 0.0556 & $0.0096(7)$ & 0.1182 \\
\hline N/Gaussian & $0.0644(47)$ & 0.0855 & 0.5678 & 0.1937 & $0.0192(14)$ & 0.0266 & $0.0123(9)$ & 0.0180 \\
\hline Univariate & $0.0288(21)$ & 0.0045 & 0.6310 & 0.0157 & $0.0082(6)$ & 0.6204 & $0.0068(5)$ & 0.5007 \\
\hline \multicolumn{9}{|c|}{ Hour 2} \\
\hline Skew $t /$ SJC & $0.0616(45)$ & 0.1604 & 0.6027 & 0.3260 & $0.0137(10)$ & 0.3396 & $0.0096(7)$ & 0.1182 \\
\hline N/Gaussian & $0.0616(45)$ & 0.1604 & 0.6027 & 0.3260 & $0.0137(10)$ & 0.3396 & $0.0110(8)$ & 0.0485 \\
\hline Univariate & $0.0301(22)$ & 0.0082 & 0.6912 & 0.0281 & $0.0123(9)$ & 0.5392 & $0.0041(3)$ & 0.7268 \\
\hline \multicolumn{9}{|c|}{ Hour 3} \\
\hline Skew $t /$ SJC & $0.0493(36)$ & 0.9389 & 0.5067 & 0.7998 & $0.0178(13)$ & 0.0556 & $0.0110(8)$ & 0.0485 \\
\hline N/Gaussian & $0.0411(30)$ & 0.2588 & 0.8203 & 0.5151 & $0.0178(13)$ & 0.0556 & $0.0123(9)$ & 0.0180 \\
\hline Univariate & $0.0342(25)$ & 0.0396 & 0.2704 & 0.0656 & $0.0096(7)$ & 0.9135 & 0.0055 (4) & 0.8544 \\
\hline \multicolumn{9}{|c|}{ Hour 4} \\
\hline Skew $t /$ SJC & $0.0644(47)$ & 0.0855 & 0.1015 & 0.0596 & $0.0205(15)$ & 0.0120 & $0.0123(9)$ & 0.0180 \\
\hline N/Gaussian & $0.0562(41)$ & 0.4480 & 0.2787 & 0.4171 & $0.0205(15)$ & 0.0120 & $0.0123(9)$ & 0.0180 \\
\hline Univariate & $0.0356(26)$ & 0.0618 & 0.0711 & 0.0343 & $0.0123(9)$ & 0.5392 & $0.0110(8)$ & 0.0485 \\
\hline \multicolumn{9}{|c|}{ Hour 5} \\
\hline Skew $t /$ SJC & $0.0521(38)$ & 0.7936 & 0.0526 & 0.1478 & $0.0164(12)$ & 0.1087 & $0.0096(7)$ & 0.1182 \\
\hline N/Gaussian & $0.0438(32)$ & 0.4403 & 0.0562 & 0.1200 & $0.0192(14)$ & 0.0266 & $0.0110(8)$ & 0.0485 \\
\hline Univariate & $0.0329(24)$ & 0.0244 & 0.0448 & 0.0106 & $0.0110(8)$ & 0.7947 & $0.0110(8)$ & 0.0485 \\
\hline \multicolumn{9}{|c|}{ Hour 6} \\
\hline Skew $t /$ SJC & $0.0397(29)$ & 0.1899 & 0.6204 & 0.2582 & $0.0096(7)$ & 0.9135 & $0.0082(5)$ & 0.5007 \\
\hline N/Gaussian & $0.0260(19)$ & 0.0011 & $\mathrm{NaN}$ & $\mathrm{NaN}$ & $0.0096(7)$ & 0.9135 & $0.0096(7)$ & 0.1182 \\
\hline Univariate & $0.0521(38)$ & 0.7936 & 0.4205 & 0.6986 & $0.0123(9)$ & 0.5392 & $0.0096(7)$ & 0.1182 \\
\hline
\end{tabular}

\begin{tabular}{|c|c|c|c|c|c|c|c|c|}
\hline & \multicolumn{6}{|c|}{ DE-DK1 } & & \\
\hline & \multicolumn{4}{|c|}{$q=0.05(36.5)$} & \multicolumn{2}{|c|}{$q=0.01(7.3)$} & \multicolumn{2}{|c|}{$q=0.005(3.65)$} \\
\hline & \multicolumn{8}{|c|}{ Hour 0} \\
\hline Skew $t / \mathrm{SJC}$ & $0.0274(20)$ & 0.0023 & 0.1141 & 0.0028 & $0.0055(4)$ & 0.1805 & $0.0055(4)$ & 0.8544 \\
\hline N/Gaussian & $0.0219(16)$ & 0.0001 & 0.0448 & 0.0001 & $0.0055(4)$ & 0.1804 & $0.0055(4)$ & 0.8544 \\
\hline Univariate & $0.0233(17)$ & 0.0002 & 0.4067 & 0.0008 & $0.0096(7)$ & 0.9135 & $0.0082(6)$ & 0.2582 \\
\hline \multicolumn{9}{|c|}{ Hour 1} \\
\hline Skew $t /$ SJC & $0.0233(17)$ & 0.0002 & 0.4067 & 0.0008 & $0.0055(4)$ & 0.3447 & $0.0027(2)$ & 0.3447 \\
\hline N/Gaussian & $0.0178(13)$ & 0.0000 & $\mathrm{NaN}$ & $\mathrm{NaN}$ & $0.0068(5)$ & 0.3662 & $0.0041(3)$ & 0.7268 \\
\hline Univariate & $0.0288(21)$ & 0.0045 & $\mathrm{NaN}$ & $\mathrm{NaN}$ & $0.0123(9)$ & 0.5392 & $0.0082(6)$ & 0.2582 \\
\hline \multicolumn{9}{|c|}{ Hour 2} \\
\hline Skew $t /$ SJC & $0.0356(26)$ & 0.0618 & $\mathrm{NaN}$ & $\mathrm{NaN}$ & $0.0082(6)$ & 0.6204 & $0.0041(3)$ & 0.7268 \\
\hline N/Gaussian & $0.0301(22)$ & 0.0082 & $\mathrm{NaN}$ & $\mathrm{NaN}$ & $0.0082(6)$ & 0.6204 & $0.0068(5)$ & 0.5007 \\
\hline Univariate & $0.0342(25)$ & 0.0396 & $\mathrm{NaN}$ & $\mathrm{NaN}$ & $0.0192(14)$ & 0.0266 & $0.0164(12)$ & 0.0005 \\
\hline \multicolumn{9}{|c|}{ Hour 3} \\
\hline Skew $t / \mathrm{SJC}$ & $0.0438(32)$ & 0.4403 & 0.7077 & 0.6921 & $0.0123(9)$ & 0.5392 & $0.0055(4)$ & 0.8544 \\
\hline N/Gaussian & $0.0370(27)$ & 0.0929 & 0.3560 & 0.1592 & $0.0123(9)$ & 0.5392 & $0.0068(5)$ & 0.5007 \\
\hline Univariate & $0.0384(28)$ & 0.1351 & $\mathrm{NaN}$ & $\mathrm{NaN}$ & $0.0205(15)$ & 0.0120 & $0.0123(9)$ & 0.0180 \\
\hline \multicolumn{9}{|c|}{ Hour 4} \\
\hline Skew $t /$ SJC & $0.0452(33)$ & 0.5516 & 0.2504 & 0.4327 & $0.0110(8)$ & 0.7947 & $0.0055(4)$ & 0.8544 \\
\hline N/Gaussian & $0.0384(28)$ & 0.1351 & 0.4034 & 0.2309 & $0.0110(8)$ & 0.7947 & $0.0096(7)$ & 0.1182 \\
\hline Univariate & $0.0534(39)$ & 0.6681 & 0.2077 & 0.4125 & $0.0192(14)$ & 0.0266 & $0.0123(9)$ & 0.0180 \\
\hline \multicolumn{9}{|c|}{ Hour 5} \\
\hline Skew $t /$ SJC & $0.0479(35)$ & 0.8041 & 0.3305 & 0.6040 & $0.0096(7)$ & 0.9135 & $0.0014(1)$ & 0.0995 \\
\hline N/Gaussian & $0.0425(31)$ & 0.3423 & 0.1835 & 0.2631 & $0.0110(8)$ & 0.7947 & $0.0027(2)$ & 0.3447 \\
\hline Univariate & $0.0452(33)$ & 0.5516 & 0.6787 & 0.7687 & $0.0192(14)$ & 0.0266 & $0.0137(10)$ & 0.0061 \\
\hline \multicolumn{9}{|c|}{ Hour 6} \\
\hline Skew $t / \mathrm{SJC}$ & $0.0260(19)$ & 0.0011 & 0.5149 & 0.0041 & $0.0055(4)$ & 0.1805 & $0.0014(1)$ & 0.0995 \\
\hline N/Gaussian & $0.0164(12)$ & 0.0000 & 0.1857 & 0.0000 & $0.0055(4)$ & 0.1805 & $0.0041(3)$ & 0.7268 \\
\hline Univariate & $0.0671(49)$ & 0.0423 & 0.1184 & 0.0376 & $0.0329(24)$ & 0.0000 & $0.0288(21)$ & 0.0000 \\
\hline
\end{tabular}

Table 9: Coverage and test results for out-of-sample day-ahead quantile forecasts of DE-DK1 and DK1-DE. The coverage results are given with number of exeedences in parenthesis, and the reported $p$-values correspond to the tests in Christoffersen (1998) for unconditional coverage (uc), independence (ind) and conditional coverage (cc). We consider the 5\%,1\% and $0.5 \%$ quantiles of the spread distributions at seven hours of the day. The results are based on an out-of-sample period of 730 observations, from 21 May 2013 to 20 May 2015 . The $p$-values in bold highlight the cases where independence and correct (un)conditional coverage are rejected at a $5 \%$ significance level. 


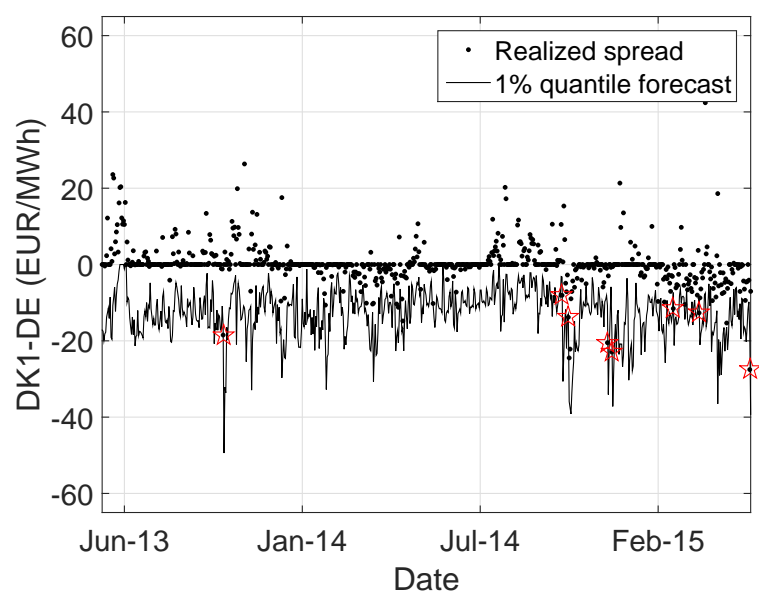

(a) DK1-DE Hour 0

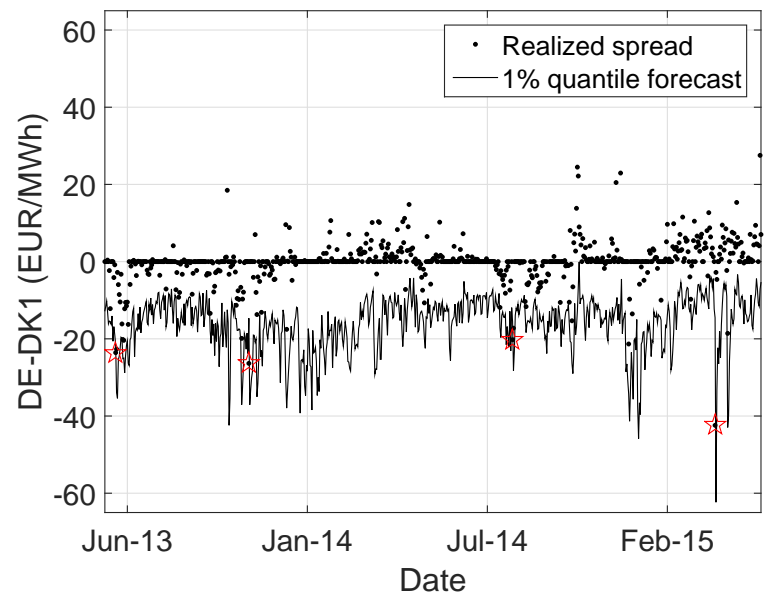

(b) DE-DK1 Hour 0

Figure 7: Out-of-sample day-ahead forecasts for the $1 \%$ quantile of spread distributions with a Skew $t /$ SJC model. The red stars signal exceedances.

model. Although not reported in Table 9, the independence test is also passed for the remaining quantile forecasts, whenever this test is defined. Thus, it is to a high extent the rejection of unconditional coverage that leads to the rejection of conditional coverage.

To provide a clearer picture of the model performance results, we report in Table 10 model acceptance percentages that are computed for different groupings: Overall, for each one of the tails, across the three quantiles, and across the seven hours of the day. For each quantile, hour of the day and tail of the spread distribution, a model is labeled as "accepted" if all three tests are passed (given that they are defined).

The results in Table 10 suggest that the Skew $t$ /SJC model performs the best, with the overall acceptance and the acceptance across tails and quantiles always being superior to the other models. As far as acceptance across the hours of the day is concerned, the Skew $t / \mathrm{SJC}$ model always outperforms the Univariate model, and performs better or at least as good as the N/Gaussian model. When comparing the N/Gaussian model with the Univariate model, we find stronger empirical evidence for the former, which performs better in all but two instances cf. Table 10. Interestingly, we also point out that while the performance of the Skew $t /$ SJC model is consistent across the two tails of the distribution, the performance of the other models is asymmetric. Specifically, the N/Gaussian performs much better when considering the $5 \%, 1 \%$ and $0.5 \%$ quantiles of the DE-DK1 distribution as opposed to the DK1-DE distribution, while the reverse is true for the Univariate model.

\begin{tabular}{|c|c|c|c|c|c|c|c|c|c|c|c|c|c|}
\hline \multirow[b]{4}{*}{ Skew $t /$ SJC } & \multicolumn{13}{|c|}{ Acceptance percentage } \\
\hline & \multirow[b]{2}{*}{ Overall } & \multirow[b]{2}{*}{ DK1-DE } & \multirow[b]{2}{*}{ DE-DK1 } & \multicolumn{3}{|c|}{ Across quantiles } & \multicolumn{7}{|c|}{ Across hours } \\
\hline & & & & 0.05 & 0.01 & 0.005 & $\mathrm{H} 0$ & H 1 & $\mathrm{H} 2$ & H 3 & $\mathrm{H} 4$ & H 5 & H 6 \\
\hline & 86 & 86 & 86 & 79 & 93 & 86 & 83 & 83 & 100 & 83 & 67 & 100 & 83 \\
\hline N/Gaussian & 69 & 57 & 81 & 64 & 79 & 64 & 83 & 50 & 67 & 83 & 67 & 67 & 67 \\
\hline Univariate & 48 & 62 & 33 & 29 & 64 & 50 & 67 & 67 & 33 & 50 & 33 & 33 & 50 \\
\hline
\end{tabular}

Table 10: Acceptance percentages based on the forecast results in Table 9. A model is labeled as "accepted" if all tests are passed, conditional on the test output being different from NaN.

Overall, the two-year out-of-sample evaluations of tail quantile forecasts for the DK1-DE pair strengthen further the previously drawn conclusions regarding the usefulness of the proposed copula framework, and the importance of capturing heavy tails, asymmetric dependence and tail dependence. The out-of-sample performance of the Skew $t /$ SJC model is reasonable when evaluated on its own, and superior when compared with the considered alternative models. While the results are encouraging, they do leave place for improvement. There are many changes and extensions to the proposed regime-switching AR-GARCH copula model that are easily implementable and may improve the tail quantile forecast performance. For example, we mention replacing GARCH with EGARCH (see Knittel and Roberts (2005) and Chan and Gray (2006) for the rationale behind this), considering even more flexible copulas, e.g. copula mixtures, and allowing the copula family to change thorough the out-of-sample forecasting period. We leave however such comparisons for future study. 


\section{Conclusion and outlook}

In this paper we propose a regime-switching AR-GARCH copula for modeling pairs of day-ahead electricity prices in interconnected markets. The regimes are observable, distinguishing the case of identical prices from the case of non-identical prices. The AR-GARCH filters account for the serial dependence in the conditional mean and the conditional variance equations, and the copula is introduced to easily relax the assumption of normal margins, and to investigate the dependency between prices beyond linear correlation.

Aside from being able to accommodate the structural change in the joint price dynamics that followed the price coupling of areas, we obtain strong in-sample evidence for the modeling framework. The same type of regime-switching AR-GARCH filter is applied to four pairs of prices and each hour of the day, a total of 96 models, with all results confirming the superiority of the skewed $t$ distribution over the normal distribution, and also whiteness of residuals. The copula analysis performed on the filtered data belonging to the non-identical price regime reveals that the characterization of the dependence structure with one single number, namely the linear correlation, is indeed not sufficient in most of the cases. In the context of copulas, we find significant evidence of tail dependence in all pairs of prices we consider. Moreover, we also find evidence of asymmetry in the dependence structure.

As a first application we consider the pricing of financial transmission rights, where the effects of heavy tails and a better fitting copula (compared to the Gaussian one) are highlighted. We find heavy tails to have a significant effect on option prices in terms of magnitude, but are of mixed sign. The moneyness of the option influences the magnitude of the effect, with out-of-the-money options being most influenced. Like in the case of heavy tails, the choice of copula model affects out-of-the-money options the most. Generally, lower option prices are produced with the preferred copula specification compared to the Gaussian one.

As a second application, the tail quantile forecast performance of three competing models is evaluated. For the forecast exercise, two of the models are copula-based, while the last model represents a much simpler and very different approach. Forecast performance is evaluated based on the likelihood ratio tests proposed in Christoffersen (1998), with the results revealing that the best performing model is a copula-based model where heavy tails, asymmetric dependence and tail dependence are incorporated.

Although major advances were made over the past years toward achieving the goal of a single European electricity market, the completion of such a project lies far out in the future. Developing models for the joint dynamics of prices in interconnected markets that comply with the present institutional framework is hence of great relevance. However, just as the price coupling of areas has led to a change in the structure of day-ahead electricity prices, ongoing developments challenge further keeping the models up-to-date. For example, recall that our sample window ends on 20 May 2015, which represents the change in market coupling algorithm for the CWE region (transitioning from the ATC to the more efficient FB methodology). We wish to briefly shed some light on implications caused by this change in the context of the future use of our proposed model. To this end, we enlarge our sample window, allowing it to end on 25 September 2016, and fit all regime-switching ARGARCH models to this data, confirming of course the goodness-of-fit of the skew $t$ distribution and whiteness of residuals. Then, we perform an analysis similar to that in Sec. 4.3, namely, we test for a break in the rank correlation at $t^{*}=20$ May 2015. Specifically, we wish to test

$$
H_{0}: \rho^{A T C}=\rho^{F B} \quad \text { vs. } \quad H_{1}: \rho^{A T C} \neq \rho^{F B},
$$

where $\rho^{A T C}$ denotes the rank correlation between filtered prices belonging to the non-identical price regime in the interval $\left[1, t^{*}\right]$, and $\rho^{F B}$ denotes the rank correlation in the interval $\left(t^{*}, T\right]$.

We apply the test to all four pairs of prices, although the change in methodology does not concern the DE-DK1 pair. The results are presented in Table 11, and show significant evidence against the null of equal rank correlations for the CWE pairs. In the case of DE-FR, the null is rejected 17 out of 24 times in favor of the alternative hypothesis. In the case of DE-NL and NL-BE, the null is rejected 8 and 12 times, respectively. As expected, there is not enough evidence for a break in the case of DE-DK1 at any hour of the day.

In terms of our modeling framework, such a break can be accommodated by e.g. retaining the same copula model, but allowing the copula dependence parameter to change between the two periods. Also interesting to notice is that the transition to the FB methodology generally implies a stronger dependence for the CWE pairs. With everything else being equal, the continuation of such developments in the future would imply that the effects of e.g. tail dependence highlighted in Sections 4.4.1, 5 and 6 will be amplified, and thus be even more important to account for. At the present time, in-depth studies of the tail dependence after the launch of the FB market coupling are not possible due to data shortage, and we leave such extensions to future research.

Finally, we mention that spot electricity prices have a special feature in the sense that a panel of hourly prices is observed over time. In this paper, we propose a model that only considers each hour of the day separately. There is however a lot of cross-sectional dependence for the hourly prices across each day, and this dependence is not accounted for. As a topic for further research, it would be interesting to consider how this cross-sectional dependence can be modeled in the context of copulas, by e.g. applying vine copulas. 


\begin{tabular}{|c|c|c|c|c|c|c|c|c|c|c|c|c|}
\hline \multirow[t]{2}{*}{ Hour } & \multicolumn{3}{|c|}{ DE-FR } & \multicolumn{3}{|c|}{ DE-NL } & \multicolumn{3}{|c|}{ NL-BE } & \multicolumn{3}{|c|}{ DE-DK1 } \\
\hline & $\hat{\rho}^{A T C}$ & $\hat{\rho}^{F B}$ & $p$-val & $\hat{\rho}^{A T C}$ & $\hat{\rho}^{F B}$ & $p$-val & $\hat{\rho}^{A T C}$ & $\hat{\rho}^{F B}$ & $p$-val & $\hat{\rho}^{A T C}$ & $\hat{\rho}^{F B}$ & $p$-val \\
\hline 0 & 0.24 & 0.39 & 0.02 & 0.21 & 0.29 & 0.22 & 0.36 & 0.42 & 0.41 & 0.38 & 0.40 & 0.72 \\
\hline 1 & 0.31 & 0.38 & 0.23 & 0.25 & 0.36 & 0.08 & 0.37 & 0.42 & 0.41 & 0.39 & 0.41 & 0.79 \\
\hline 2 & 0.32 & 0.40 & 0.25 & 0.28 & 0.26 & 0.77 & 0.40 & 0.52 & 0.07 & 0.45 & 0.43 & 0.74 \\
\hline 3 & 0.34 & 0.51 & 0.01 & 0.27 & 0.38 & 0.08 & 0.33 & 0.50 & 0.02 & 0.48 & 0.46 & 0.71 \\
\hline 4 & 0.39 & 0.56 & 0.01 & 0.23 & 0.44 & 0.00 & 0.31 & 0.51 & 0.01 & 0.43 & 0.48 & 0.40 \\
\hline 5 & 0.35 & 0.48 & 0.09 & 0.25 & 0.43 & 0.02 & 0.32 & 0.55 & 0.00 & 0.39 & 0.48 & 0.18 \\
\hline 6 & 0.30 & 0.49 & 0.01 & 0.32 & 0.46 & 0.07 & 0.36 & 0.53 & 0.02 & 0.38 & 0.38 & 0.99 \\
\hline 7 & 0.34 & 0.62 & 0.00 & 0.39 & 0.48 & 0.22 & 0.37 & 0.44 & 0.32 & 0.40 & 0.43 & 0.53 \\
\hline 8 & 0.35 & 0.59 & 0.00 & 0.39 & 0.52 & 0.05 & 0.41 & 0.46 & 0.51 & 0.44 & 0.47 & 0.44 \\
\hline 9 & 0.27 & 0.54 & 0.00 & 0.27 & 0.41 & 0.02 & 0.44 & 0.54 & 0.14 & 0.46 & 0.48 & 0.68 \\
\hline 10 & 0.30 & 0.47 & 0.00 & 0.24 & 0.35 & 0.08 & 0.38 & 0.50 & 0.07 & 0.50 & 0.41 & 0.09 \\
\hline 11 & 0.29 & 0.48 & 0.00 & 0.20 & 0.28 & 0.19 & 0.34 & 0.53 & 0.00 & 0.48 & 0.40 & 0.17 \\
\hline 12 & 0.29 & 0.42 & 0.03 & 0.21 & 0.21 & 0.99 & 0.33 & 0.53 & 0.00 & 0.47 & 0.40 & 0.23 \\
\hline 13 & 0.31 & 0.45 & 0.02 & 0.24 & 0.14 & 0.08 & 0.32 & 0.57 & 0.00 & 0.48 & 0.44 & 0.50 \\
\hline 14 & 0.31 & 0.42 & 0.06 & 0.21 & 0.15 & 0.34 & 0.37 & 0.51 & 0.03 & 0.44 & 0.38 & 0.29 \\
\hline 15 & 0.33 & 0.49 & 0.01 & 0.22 & 0.22 & 0.99 & 0.32 & 0.56 & 0.00 & 0.43 & 0.41 & 0.66 \\
\hline 16 & 0.36 & 0.48 & 0.07 & 0.21 & 0.24 & 0.63 & 0.28 & 0.57 & 0.00 & 0.42 & 0.39 & 0.62 \\
\hline 17 & 0.36 & 0.49 & 0.07 & 0.20 & 0.30 & 0.15 & 0.25 & 0.52 & 0.00 & 0.41 & 0.41 & 0.99 \\
\hline 18 & 0.37 & 0.55 & 0.01 & 0.25 & 0.46 & 0.01 & 0.39 & 0.44 & 0.42 & 0.42 & 0.43 & 0.84 \\
\hline 19 & 0.33 & 0.61 & 0.00 & 0.27 & 0.51 & 0.00 & 0.36 & 0.46 & 0.17 & 0.36 & 0.34 & 0.76 \\
\hline 20 & 0.31 & 0.51 & 0.00 & 0.17 & 0.47 & 0.00 & 0.28 & 0.35 & 0.37 & 0.34 & 0.33 & 0.81 \\
\hline 21 & 0.35 & 0.45 & 0.07 & 0.16 & 0.43 & 0.00 & 0.33 & 0.47 & 0.04 & 0.37 & 0.30 & 0.18 \\
\hline 22 & 0.26 & 0.39 & 0.04 & 0.15 & 0.25 & 0.14 & 0.31 & 0.36 & 0.41 & 0.35 & 0.30 & 0.33 \\
\hline 23 & 0.24 & 0.37 & 0.03 & 0.21 & 0.23 & 0.65 & 0.38 & 0.44 & 0.32 & 0.33 & 0.28 & 0.41 \\
\hline
\end{tabular}

Table 11: Test results for a one time break in the rank correlations. $p$-values in bold correspond to cases where the null of no break in the rank correlation is rejected at a $5 \%$ significance level.

\section{A Additional figures regarding the fit of the models}

\section{A.1 Order of marginal models}

\begin{tabular}{lrrrr}
\hline Hour & & \multicolumn{2}{c}{ Order of marginal models } \\
& DE-FR & DE-NL & NL-BE & DE-DK1 \\
\hline 0 & $P=5, Q=0$ & $P=4, Q=0$ & $P=6, Q=0$ & $P=5, Q=1$ \\
1 & $P=3, Q=0$ & $P=4, Q=0$ & $P=6, Q=0$ & $P=5, Q=0$ \\
2 & $P=3, Q=0$ & $P=6, Q=0$ & $P=6, Q=0$ & $P=5, Q=1$ \\
3 & $P=3, Q=0$ & $P=6, Q=0$ & $P=6, Q=0$ & $P=5, Q=1$ \\
4 & $P=3, Q=0$ & $P=6, Q=0$ & $P=6, Q=0$ & $P=5, Q=0$ \\
5 & $P=3, Q=0$ & $P=6, Q=0$ & $P=6, Q=0$ & $P=4, Q=1$ \\
6 & $P=6, Q=0$ & $P=6, Q=0$ & $P=7, Q=0$ & $P=7, Q=1$ \\
7 & $P=6, Q=0$ & $P=6, Q=0$ & $P=6, Q=0$ & $P=6, Q=0$ \\
8 & $P=6, Q=0$ & $P=7, Q=0$ & $P=6, Q=0$ & $P=6, Q=0$ \\
9 & $P=7, Q=0$ & $P=6, Q=0$ & $P=6, Q=0$ & $P=6, Q=0$ \\
10 & $P=7, Q=0$ & $P=7, Q=0$ & $P=7, Q=0$ & $P=3, Q=0$ \\
11 & $P=7, Q=0$ & $P=7, Q=0$ & $P=7, Q=0$ & $P=3, Q=0$ \\
12 & $P=4, Q=0$ & $P=7, Q=0$ & $P=6, Q=0$ & $P=6, Q=0$ \\
13 & $P=6, Q=0$ & $P=7, Q=0$ & $P=7, Q=0$ & $P=6, Q=0$ \\
14 & $P=7, Q=0$ & $P=7, Q=0$ & $P=7, Q=0$ & $P=6, Q=0$ \\
15 & $P=7, Q=0$ & $P=7, Q=0$ & $P=6, Q=0$ & $P=7, Q=0$ \\
16 & $P=7, Q=0$ & $P=6, Q=0$ & $P=7, Q=0$ & $P=7, Q=0$ \\
17 & $P=7, Q=0$ & $P=7, Q=0$ & $P=7, Q=0$ & $P=6, Q=0$ \\
18 & $P=6, Q=0$ & $P=6, Q=0$ & $P=7, Q=1$ & $P=7, Q=0$ \\
19 & $P=6, Q=0$ & $P=6, Q=0$ & $P=7, Q=1$ & $P=6, Q=0$ \\
20 & $P=6, Q=0$ & $P=6, Q=0$ & $P=6, Q=0$ & $P=6, Q=0$ \\
21 & $P=6, Q=0$ & $P=6, Q=0$ & $P=7, Q=0$ & $P=6, Q=0$ \\
22 & $P=3, Q=0$ & $P=4, Q=0$ & $P=6, Q=0$ & $P=6, Q=0$ \\
23 & $P=3, Q=0$ & $P=3, Q=0$ & $P=6, Q=0$ & $P=4, Q=0$ \\
\hline & & & &
\end{tabular}

Table 12: Selected order for all marginal models based on BIC. The variable $P$ refers to the order of autoregression and the variable $Q$ refers to the order of cross-equation effects, cf. Eqs. (1) and (4). 


\section{A.2 Model check for selected models}

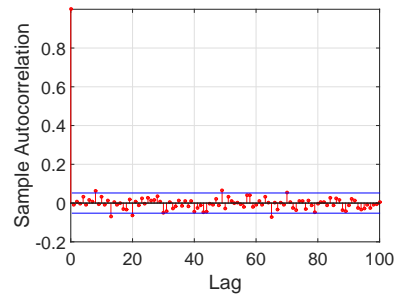

(a) DE - acf for std. residuals

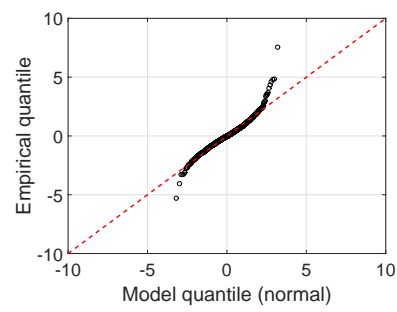

(e) DE - qq plot normal

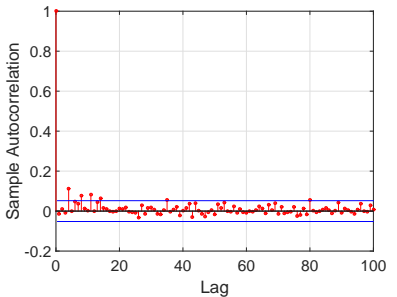

(b) DE - acf for squared std. residuals

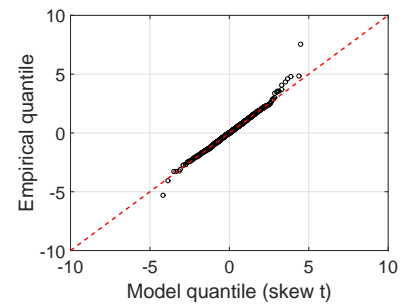

(f) DE - qq plot skew $t$

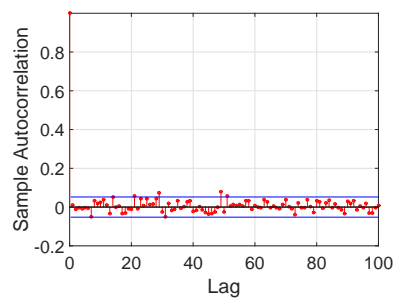

(c) NL - acf for std. residuals

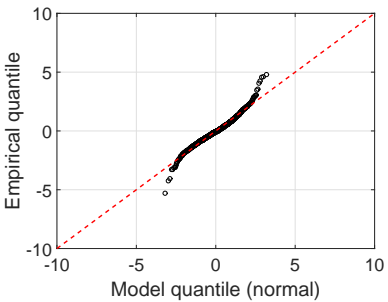

(g) NL - qq plot normal

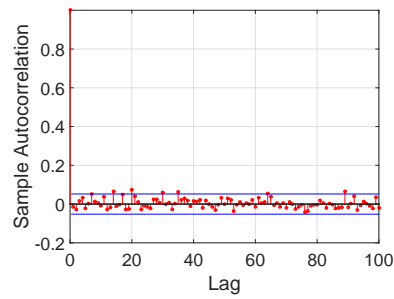

(d) NL - acf for squared std. residuals

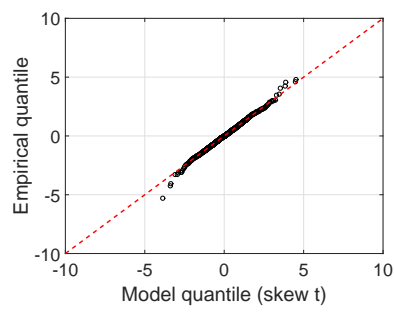

(h) NL - qq plot skew $t$

Figure 8: Sample autocorrelations and quantile plots of standardized residuals resulting from fitting regimeswitching AR-GARCH models to the DE-NL pair for hour 8.

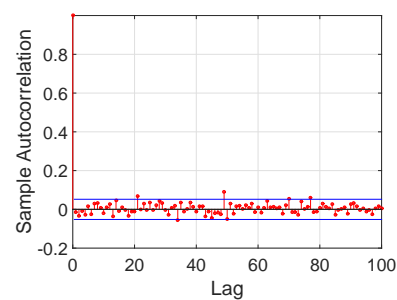

(a) NL - acf for std. residuals

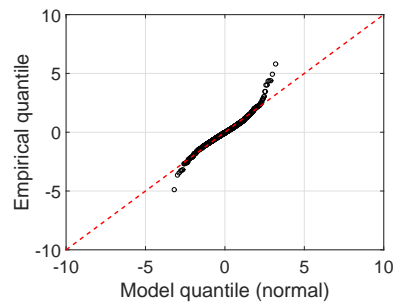

(e) NL - qq plot normal

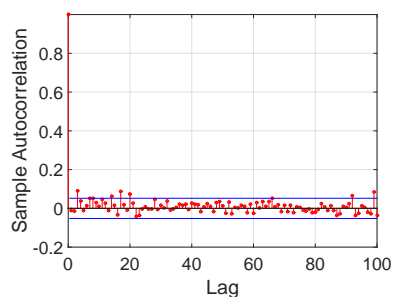

(b) NL - acf for squared std. residuals

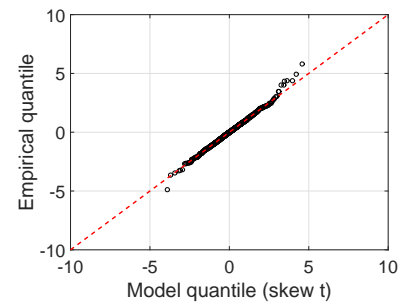

(f) NL - qq plot skew $t$

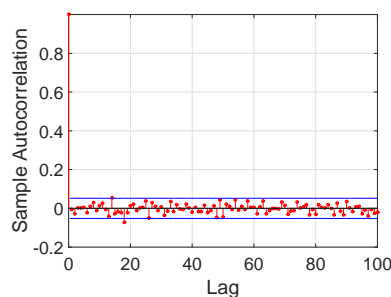

(c) BE - acf for std. residuals

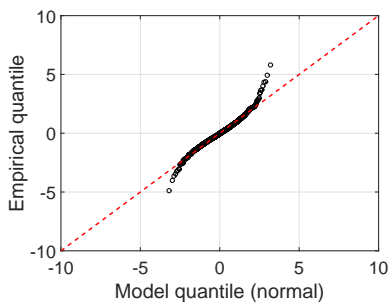

(g) BE - qq plot normal

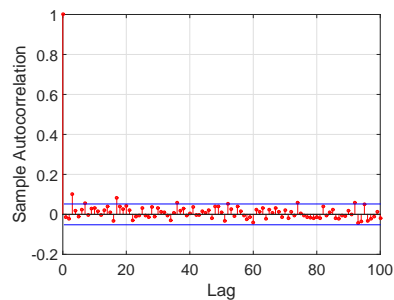

(d) BE - acf for squared std. residuals

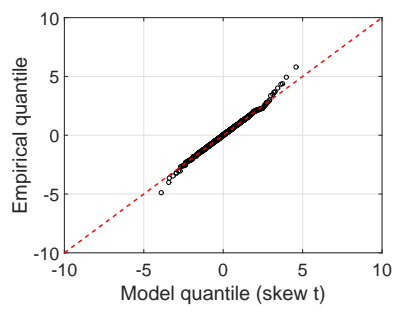

(h) BE - qq plot skew $t$

Figure 9: Sample autocorrelations and quantile plots of standardized residuals resulting from fitting regimeswitching AR-GARCH models to the NL-BE pair for hour 8. 


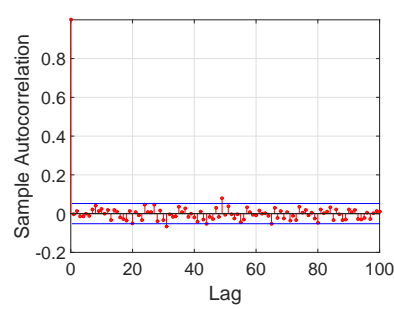

(a) DE - acf for std. residuals

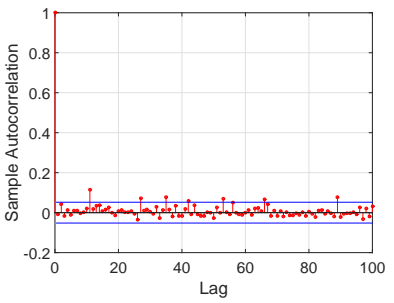

(b) DE - acf for squared std. residuals

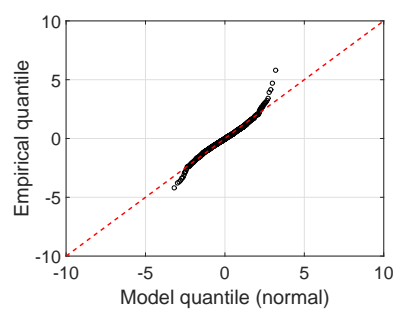

(e) DE - qq plot normal

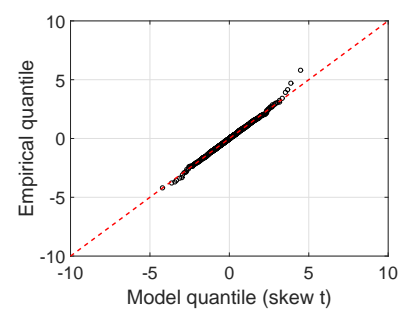

(f) DE - qq plot skew $t$

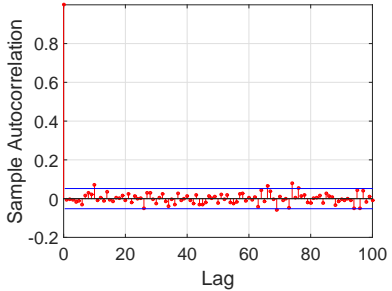

(c) DK1 - acf for std. residuals (d) DK1 - acf for squared std. residuals
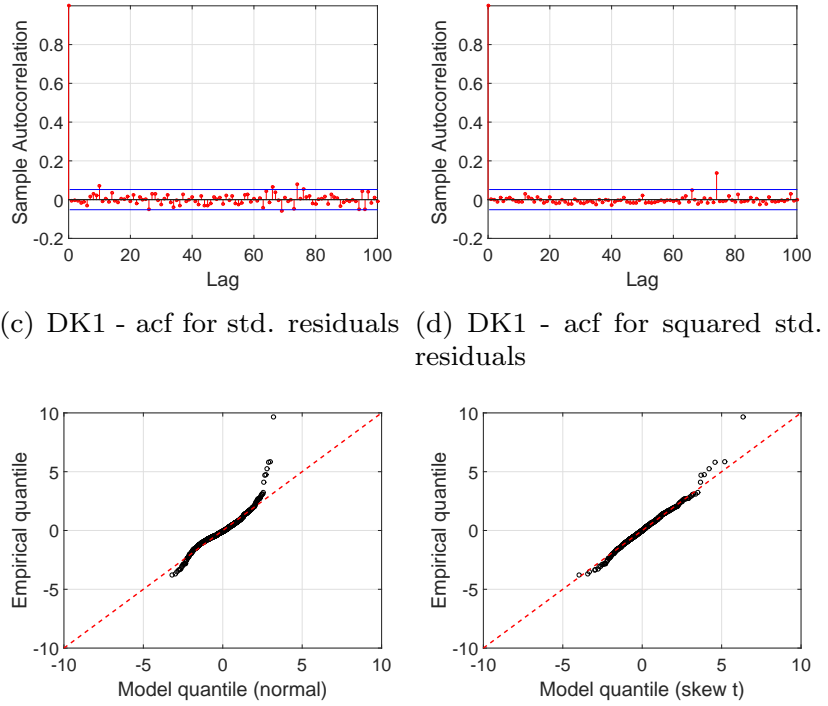

(g) DK1 - qq plot normal

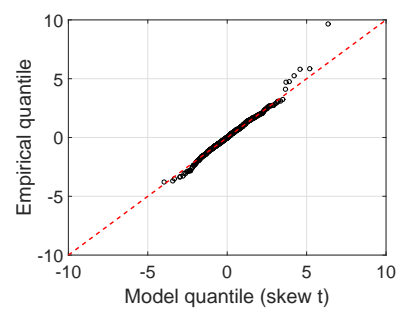

(h) DK1 - qq plot skew $t$

Figure 10: Sample autocorrelations and quantile plots of standardized residuals resulting from fitting regimeswitching AR-GARCH models to the DE-DK1 pair for hour 8.

\section{A.3 Parameter estimates for selected models}




\begin{tabular}{|c|c|c|c|c|c|c|c|c|c|}
\hline \multicolumn{10}{|c|}{ Model for the pair DE-NL hour 8} \\
\hline \multicolumn{3}{|c|}{ Equal price regime $\left(s_{t}=0\right)$} & \multicolumn{7}{|c|}{ Non-equal price regime $\left(s_{t}=1\right)$} \\
\hline & & & \multicolumn{4}{|c|}{ Area 1: DE } & \multicolumn{3}{|c|}{ Area 2: NL } \\
\hline \multicolumn{10}{|c|}{ Conditional mean (optimal order $P=7, Q=0$ ) } \\
\hline $\begin{array}{l}\hat{\phi}_{1}^{(0)} \\
\hat{\phi}_{2}^{(0)} \\
\hat{\phi}_{3}^{(0)} \\
\hat{\phi}_{4}^{(0)} \\
\hat{\phi}_{5}^{(0)} \\
\hat{\phi}_{6}^{(0)} \\
\hat{\phi}_{7}^{(0)}\end{array}$ & $\begin{array}{l}0.3451 \\
0.1753 \\
0.0588 \\
0.0588 \\
0.0162 \\
0.1306 \\
0.0898\end{array}$ & $\begin{array}{l}(0.0424) \\
(0.0419) \\
(0.0381) \\
(0.0374) \\
(0.0362) \\
(0.0345) \\
(0.0340)\end{array}$ & $\begin{array}{l}\hat{\phi}_{1}^{(1)} \\
\hat{\phi}_{2}^{(1)} \\
\hat{\phi}_{3}^{(1)} \\
\hat{\phi}_{4}^{(1)} \\
\hat{\phi}_{5}^{(1)} \\
\hat{\phi}_{6}^{(1)} \\
\hat{\phi}_{7}^{(1)}\end{array}$ & $\begin{array}{r}0.2923 \\
0.0905 \\
-0.0197 \\
0.0277 \\
0.0073 \\
0.1279 \\
0.0454\end{array}$ & $\begin{array}{l}(0.0573) \\
(0.0573) \\
(0.0571) \\
(0.0515) \\
(0.0516) \\
(0.0516) \\
(0.0481)\end{array}$ & & $\begin{array}{l}\hat{\phi}_{1}^{(2)} \\
\hat{\phi}_{2}^{(2)} \\
\hat{\phi}_{3}^{(2)} \\
\hat{\phi}_{4}^{(2)} \\
\hat{\phi}_{5}^{(2)} \\
\hat{\phi}_{6}^{(2)} \\
\hat{\phi}_{7}^{(2)}\end{array}$ & $\begin{array}{r}0.2419 \\
0.1469 \\
-0.0581 \\
0.0950 \\
0.0093 \\
0.1192 \\
0.1195\end{array}$ & $\begin{array}{l}(0.0517) \\
(0.0507) \\
(0.0501) \\
(0.0493) \\
(0.0482) \\
(0.0468) \\
(0.0447)\end{array}$ \\
\hline \multicolumn{10}{|c|}{ Conditional variance } \\
\hline $\begin{array}{l}\hat{\omega}^{(0)} \\
\hat{\alpha}^{(0)} \\
\hat{\beta}^{(0)}\end{array}$ & $\begin{array}{l}9.2195 \\
0.1655 \\
0.5524\end{array}$ & $\begin{array}{l}(4.6335) \\
(0.0672) \\
(0.1299)\end{array}$ & $\begin{array}{l}\hat{\omega}^{(1)} \\
\hat{\alpha}^{(1)} \\
\hat{\beta}^{(1)}\end{array}$ & $\begin{array}{r}36.7656 \\
0.2444 \\
0.4139\end{array}$ & $\begin{array}{r}(13.7786) \\
(0.1480) \\
(0.2149)\end{array}$ & & $\begin{array}{l}\hat{\omega}^{(2)} \\
\hat{\alpha}^{(2)} \\
\hat{\beta}^{(2)}\end{array}$ & $\begin{array}{l}8.6863 \\
0.1514 \\
0.7432\end{array}$ & $\begin{array}{l}(7.5150) \\
(0.0991) \\
(0.1795)\end{array}$ \\
\hline \multicolumn{10}{|c|}{ Marginal distribution (skew $t$ ) } \\
\hline $\begin{array}{l}\hat{\nu}^{(0)} \\
\hat{\lambda}^{(0)}\end{array}$ & $\begin{array}{l}5.7397 \\
0.0847\end{array}$ & $\begin{array}{l}(1.6234) \\
(0.0431)\end{array}$ & $\begin{array}{l}\hat{\nu}^{(1)} \\
\hat{\lambda}^{(1)}\end{array}$ & $\begin{array}{l}4.4073 \\
0.0248\end{array}$ & $\begin{array}{l}(1.0694) \\
(0.0518)\end{array}$ & & $\begin{array}{l}\hat{\nu}^{(2)} \\
\hat{\lambda}^{(2)}\end{array}$ & $\begin{array}{l}5.4083 \\
0.1642\end{array}$ & $\begin{array}{l}(1.5802) \\
(0.0514)\end{array}$ \\
\hline & & & \multicolumn{7}{|c|}{ Optimal copula model (SJC) } \\
\hline & & & & & $\begin{array}{l}\hat{\tau}^{L} \\
\hat{\tau}^{U}\end{array}$ & $\begin{array}{l}0.1605 \\
0.3189\end{array}$ & $\begin{array}{l}(0.0569) \\
(0.0496)\end{array}$ & & \\
\hline
\end{tabular}

Table 13: Parameter estimates for the DE-NL hour 8 model. Simulation based standard errors are given in parenthesis, and are based on 999 simulations.

\begin{tabular}{|c|c|c|c|c|c|c|c|c|c|}
\hline \multicolumn{10}{|c|}{ Model for the pair NL-BE hour 8} \\
\hline \multicolumn{3}{|c|}{ Equal price regime $\left(s_{t}=0\right)$} & \multicolumn{7}{|c|}{ Non-equal price regime $\left(s_{t}=1\right)$} \\
\hline & & & \multicolumn{3}{|c|}{ Area 1: NL } & & \multicolumn{3}{|c|}{ Area 2: $\mathrm{BE}$} \\
\hline \multicolumn{10}{|c|}{ Conditional mean (optimal order $P=6, Q=0$ ) } \\
\hline$\hat{\phi}_{1}^{(0)}$ & 0.3690 & $(0.0376)$ & $\hat{\phi}_{1}^{(1)}$ & 0.3421 & $(0.0700)$ & & $\hat{\phi}_{1}^{(2)}$ & 0.3533 & $(0.0698)$ \\
\hline$\hat{\phi}_{2}^{(0)}$ & 0.1643 & $(0.0369)$ & $\hat{\phi}_{2}^{(1)}$ & 0.2277 & $(0.0704)$ & & $\hat{\phi}_{2}^{(2)}$ & 0.1006 & $(0.0633)$ \\
\hline$\hat{\phi}_{3}^{(0)}$ & 0.1028 & $(0.0350)$ & $\hat{\phi}_{3}^{(1)}$ & -0.0410 & $(0.0731)$ & & $\hat{\phi}_{3}^{(2)}$ & 0.1039 & $(0.0600)$ \\
\hline$\hat{\phi}_{4}^{(0)}$ & 0.0474 & $(0.0321)$ & $\hat{\phi}_{4}^{(1)}$ & -0.0302 & $(0.0665)$ & & $\hat{\phi}_{4}^{(2)}$ & 0.1308 & $(0.0536)$ \\
\hline$\hat{\phi}_{5}^{(0)}$ & -0.0182 & $(0.0303)$ & $\hat{\phi}_{5}^{(1)}$ & 0.1502 & $(0.0648)$ & & $\hat{\phi}_{5}^{(2)}$ & 0.0374 & $(0.0526)$ \\
\hline$\hat{\phi}_{6}^{(0)}$ & 0.1561 & $(0.0279)$ & $\hat{\phi}_{6}^{(1)}$ & 0.2186 & $(0.0648)$ & & $\hat{\phi}_{6}^{(2)}$ & 0.1237 & $(0.0504)$ \\
\hline \multicolumn{10}{|c|}{ Conditional variance } \\
\hline$\hat{\omega}^{(0)}$ & 15.7739 & $(4.6886)$ & $\hat{\omega}^{(1)}$ & 82.7166 & $(15.1057)$ & & $\hat{\omega}^{(2)}$ & 23.0052 & $(9.8562)$ \\
\hline$\hat{\alpha}^{(0)}$ & 0.2673 & $(0.0769)$ & $\hat{\alpha}^{(1)}$ & 0.1863 & $(0.1492)$ & & $\hat{\alpha}^{(2)}$ & 0.4751 & $(0.1965)$ \\
\hline$\hat{\beta}^{(0)}$ & 0.3470 & $(0.1157)$ & $\hat{\beta}^{(1)}$ & 0.1467 & $(0.1932)$ & & $\hat{\beta}^{(2)}$ & 0.2754 & $(0.2018)$ \\
\hline \multicolumn{10}{|c|}{ Marginal distribution (skew $t$ ) } \\
\hline$\hat{\nu}^{(0)}$ & 6.1638 & $(1.6688)$ & $\hat{\nu}^{(1)}$ & 6.0508 & $(3.1611)$ & & $\hat{\nu}^{(2)}$ & 4.1789 & $(1.3552)$ \\
\hline \multirow[t]{4}{*}{$\hat{\lambda}^{(0)}$} & 0.0946 & $(0.0400)$ & $\hat{\lambda}^{(1)}$ & 0.0561 & $(0.0661)$ & & $\hat{\lambda}^{(2)}$ & 0.0706 & $(0.0599)$ \\
\hline & & & \multicolumn{7}{|c|}{ Optimal copula model (SJC) } \\
\hline & & & & & $\hat{\tau}^{L}$ & 0.2121 & $(0.0681)$ & & \\
\hline & & & & & $\hat{\tau}^{U}$ & 0.2963 & $(0.0646)$ & & \\
\hline
\end{tabular}

Table 14: Parameter estimates for the NL-BE hour 8 model. Simulation based standard errors are given in parenthesis, and are based on 999 simulations. 


\begin{tabular}{|c|c|c|c|c|c|c|c|c|c|}
\hline \multicolumn{10}{|c|}{ Model for the pair DE-DK1 hour 8} \\
\hline \multicolumn{3}{|c|}{ Equal price regime $\left(s_{t}=0\right)$} & \multicolumn{7}{|c|}{ Non-equal price regime $\left(s_{t}=1\right)$} \\
\hline & & & \multicolumn{3}{|c|}{ Area 1: DE } & & \multicolumn{3}{|c|}{ Area 2: DK1 } \\
\hline \multicolumn{10}{|c|}{ Conditional mean (optimal order $P=6, Q=0$ ) } \\
\hline$\hat{\phi}_{1}^{(0)}$ & 0.3678 & $(0.0605)$ & $\hat{\phi}_{1}^{(1)}$ & 0.3419 & $(0.0381)$ & & $\hat{\phi}_{1}^{(2)}$ & 0.3709 & $(0.0434)$ \\
\hline$\hat{\phi}_{2}^{(0)}$ & 0.1709 & $(0.0664)$ & $\hat{\phi}_{2}^{(1)}$ & 0.1432 & $(0.0366)$ & & $\hat{\phi}_{2}^{(2)}$ & 0.0463 & $(0.0407)$ \\
\hline$\hat{\phi}_{3}^{(0)}$ & 0.0586 & $(0.0624)$ & $\hat{\phi}_{3}^{(1)}$ & 0.0721 & $(0.0392)$ & & $\hat{\phi}_{3}^{(2)}$ & 0.0854 & $(0.0372)$ \\
\hline$\hat{\phi}_{4}^{(0)}$ & 0.0242 & $(0.0602)$ & $\hat{\phi}_{4}^{(1)}$ & 0.0226 & $(0.0368)$ & & $\hat{\phi}_{4}^{(2)}$ & 0.0338 & $(0.0381)$ \\
\hline$\hat{\phi}_{5}^{(0)}$ & 0.0862 & $(0.0574)$ & $\hat{\phi}_{5}^{(1)}$ & 0.0054 & $(0.0375)$ & & $\hat{\phi}_{5}^{(2)}$ & 0.0426 & $(0.0369)$ \\
\hline$\hat{\phi}_{6}^{(0)}$ & 0.1507 & $(0.0540)$ & $\hat{\phi}_{6}^{(1)}$ & 0.1094 & $(0.0351)$ & & $\hat{\phi}_{6}^{(2)}$ & 0.0792 & $(0.0446)$ \\
\hline \multicolumn{10}{|c|}{ Conditional variance } \\
\hline$\hat{\omega}^{(0)}$ & 0.0008 & (10.1640) & $\hat{\omega}^{(1)}$ & 4.2682 & $(3.8344)$ & & $\hat{\omega}^{(2)}$ & 70.2888 & (20.4931) \\
\hline$\hat{\alpha}^{(0)}$ & 0.1323 & $(0.0718)$ & $\hat{\alpha}^{(1)}$ & 0.1811 & $(0.0556)$ & & $\hat{\alpha}^{(2)}$ & 0.1715 & $(0.1159)$ \\
\hline$\hat{\beta}^{(0)}$ & 0.8325 & $(0.1479)$ & $\hat{\beta}^{(1)}$ & 0.7515 & $(0.0826)$ & & $\hat{\beta}^{(2)}$ & 0.1856 & $(0.2307)$ \\
\hline \multicolumn{10}{|c|}{ Marginal distribution (skew $t$ ) } \\
\hline$\hat{\nu}^{(0)}$ & 6.5004 & $(3.5006)$ & $\hat{\nu}^{(1)}$ & 5.7861 & $(1.2491)$ & & $\hat{\nu}^{(2)}$ & 3.7827 & $(0.5552)$ \\
\hline \multirow[t]{3}{*}{$\hat{\lambda}^{(0)}$} & 0.0417 & $(0.0644)$ & $\hat{\lambda}^{(1)}$ & 0.0368 & $(0.0424)$ & & $\hat{\lambda}^{(2)}$ & 0.2301 & $(0.0358)$ \\
\hline & & & \multicolumn{7}{|c|}{ Optimal copula model (Student $t$ ) } \\
\hline & & & & & $\hat{\rho}$ & $\begin{array}{r}0.4678 \\
125189\end{array}$ & 267) & & \\
\hline
\end{tabular}

Table 15: Parameter estimates for the DE-DK1 hour 8 model. Simulation based standard errors are given in parenthesis, and are based on 999 simulations.

\section{References}

Aas, K., Czado, C., Frigessi, A. and Bakken, H., 2009. Pair-copula constructions of multiple dependence. Insurance: Mathematics and Economics, 44(2), 182-198.

Avdulaj, K. and Barunikl, J., 2015. Are benefits from oil - stocks diversification gone? New evidence from a dynamic copula and high frequency data. Energy Economics, 51, 31-44.

Benth, F.E., Šaltytė Benth, J. and Koekebakker, S., 2008. Stochastic Modeling of Electricity and Related Markets. World Scientific.

Benth, F.E. and Meyer-Brandis, T., 2009. The information premium for non-storable commodities. Journal of Energy Markets, 2(3), 111-140.

Benth, F.E. and Kettler, P.C., 2011. Dynamic copula models for the spark spread. Quantitative Finance, 11(3), 407-421.

Benth, F.E. and Schmeck, M.D., 2014. Pricing futures and options in electricity markets. In The Interrelationship Between Financial and Energy Markets, edited by S. Ramos and H. Veiga, Lecture Notes in Energy 54, 233260, Springer Verlag.

Burger, M., Klar, B., Muller, A. and Schindlmayr, G., 2004. A spot market model for pricing derivatives in electricity markets. Quantitative Finance, 4, 109-122.

Börger, R., Cartea, A., Kiesel, R. and Schindlmayr, G., 2009. Cross-commodity analysis and applications to risk management. Journal of Futures Markets, 29(3), 197-217.

Carmona, R., Coulon, M. and Schwarz, D., 2013. Electricity price modeling and asset valuation: A multi-fuel approach. Math. Financ. Econ., 7(2), 167-202.

Chan, K. F. and Gray, P., 2006. Using extreme value theory to measure value-at-risk for daily electricity spot prices. International Journal of Forecasting, 22, 283-300.

Christoffersen, P.F., 1998. Evaluating interval forecasts. International Economics Review, 39(4), 841-862.

Dias, A. and Embrechts, P., 2009. Testing for structural changes in exchange rates dependence beyond linear correlation. The European Journal of Finance, 15(7-8), 619-637. 
Elberg, C. and Hagspiel, S., 2015. Spatial dependencies of wind power and interrelations with spot price dynamics. European Journal of Operational Research, 241(1), 260-272.

EPEX, 2015. Flow-Based methodology for CWE market coupling successfully launched.

Füss, R., Mahringer, S. and Prokopczuk, M., 2013. Electricity spot and derivatives pricing when markets are interconnected. University of St. Gallen, School of Finance Research Paper No. 1323.

Füss, R., Mahringer, S. and Prokopczuk, M., 2015. Electricity market coupling and the pricing of transmission rights: An option-based approach. University of St. Gallen, School of Finance Research Paper No. 1512.

Grothe, O. and Schnieders, J., 2011. Spatial dependence in wind and optimal wind power allocation: A copulabased analysis. Energy Policy, 39(9), 4742-4754.

Haldrup, N. and Nielsen, M.Ø., 2006. A regime switching long memory model for electricity prices. Journal of Econometrics, 135(1-2), 349-376.

Haldrup, N., Nielsen, F.S. and Nielsen, M.Ø., 2010. A vector autoregressive model for electricity prices subject to long memory and regime switching. Energy Economics, 32(5), 1044-1058.

Janczura, J., Trück, S., Weron, R. and Wolff, R.C., 2013. Identifying spikes and seasonal components in electricity spot price data: A guide to robust modeling. Energy Economics, 38, 96-110.

Joe, H., 1997. Multivariate Models and Dependence Concepts. 1st Edition, Chapman and Hall.

Keles, D., Genoese, M., Möst, D. and Fichtner, W., 2012. Comparison of extended mean-reversion and time series models for electricity spot price simulation considering negative prices. Energy Economics, 34(4), 10121032.

Kiesel, R. and Kustermann, M., 2016. Structural models for coupled electricity markets. Journal of Commodity Markets, 3(1), 16-38.

Knittel, C. R. and Roberts, M. R., 2005. An empirical examination of restructured electricity prices. Energy Economics, 27, 791-817.

Lucia, J.J. and Schwartz, E.S., 2002. Electricity prices and power derivatives: Evidence from the Nordic Power Exchange. Review of Derivatives Research, 5(1), 5-50.

Nelsen, R.B., 1999. An Introduction to Copulas. Springer.

Paraschiv, F., 2013. Price dynamics in electricity markets. In Handbook of Risk Management in Energy Production and Trading, edited by R. M. Kovacevic, G. Ch. Pflug and M. T. Vespucci, 47-69, Springer.

Patton, A.J., 2001. Modelling time-varying exchange rate dependence using the conditional copula. UCSD Dept. of Economics, working paper.

Patton, A.J., 2006. Modelling asymmetric exchange rate dependence. International Economic Review, 47(2), $527-556$.

Patton, A.J., 2013. Copula methods for forecasting multivariate time series. In Handbook of Economic Forecasting, edited by G. Elliott and A. Timmermann, Vol. 2B, 899-960, Elsevier B.V.

Sklar, A., 1959. Fonctions de répartition à n dimensions et leurs marges. Publications de l'Institut de Statistique de L'Université de Paris, 8, 229-231.

TenneT, 2013. Market review 2013. 